Article

\title{
Activation of Cdc42 GTPase upon CRY2-Induced Cortical Recruitment Is Antagonized by GAPs in Fission Yeast
}

\author{
Iker Lamas ${ }^{\circledR}$, Nathalie Weber and Sophie G. Martin *(1) \\ Department of Fundamental Microbiology, Faculty of Biology and Medicine, University of Lausanne, Biophore \\ building, 1015 Lausanne, Switzerland; iker.lamasherrera@unil.ch (I.L.); nadliweber@hotmail.com (N.W.) \\ * Correspondence: Sophie.Martin@unil.ch
}

Received: 2 June 2020; Accepted: 7 September 2020; Published: 12 September 2020

check for updates

\begin{abstract}
The small GTPase Cdc42 is critical for cell polarization in eukaryotic cells. In rod-shaped fission yeast Schizosaccharomyces pombe cells, active GTP-bound Cdc42 promotes polarized growth at cell poles, while inactive Cdc42-GDP localizes ubiquitously also along cell sides. Zones of Cdc42 activity are maintained by positive feedback amplification involving the formation of a complex between Cdc42-GTP, the scaffold Scd2, and the guanine nucleotide exchange factor (GEF) Scd1, which promotes the activation of more Cdc42. Here, we use the CRY2-CIB1 optogenetic system to recruit and cluster a cytosolic $\mathrm{Cdc} 42$ variant at the plasma membrane and show that this leads to its moderate activation also on cell sides. Surprisingly, Scd2, which binds Cdc42-GTP, is still recruited to CRY2-Cdc42 clusters at cell sides in individual deletion of the GEFs Scd1 or Gef1. We show that activated Cdc42 clusters at cell sides are able to recruit Scd1, dependent on the scaffold Scd2. However, Cdc42 activity is not amplified by positive feedback and does not lead to morphogenetic changes, due to antagonistic activity of the GTPase activating protein Rga4. Thus, the cell architecture is robust to moderate activation of $\mathrm{Cdc} 42$ at cell sides.
\end{abstract}

Keywords: Cdc42; GTPase activating protein (GAP); cell polarity; fission yeast Schizosaccharomyces pombe; CRY2-CIBN; optogenetics; clustering; positive feedback; pattern formation

\section{Introduction}

In eukaryotes, the small Rho-family GTPase Cdc42 is a highly conserved regulator of cell morphogenesis, proliferation, and differentiation. Prenylation of Cdc42's C-terminal CAAX motif underlies its association with the plasma membrane, where it functions as a molecular switch that alternates between GTP-bound, active and GDP-bound, inactive states. Activation of Rho GTPases relies on the activity of guanine nucleotide exchange factors (GEFs), while their intrinsic GTPase activity is enhanced by GTPase activating proteins (GAPs) to return them to the inactive state. GDP-bound Cdc42 also binds guanine-nucleotide dissociation inhibitors (GDI), which both block the exchange of GDP by GTP and solubilize Cdc42-GDP in the cytosol [1-3].

In the fission yeast Schizosaccharomyces pombe, $\mathrm{Cdc} 42$ is active at sites of polarized growth during vegetative and sexual life cycles. GTP-loading is promoted by two GEFs, Scd1 and Gef1. Scd1, which localizes to cell poles, receives information from the upstream Ras1 GTPase signal and mediates feedback control through the scaffolding activity of Scd2 [4-6]. For this, Scd1 forms a quaternary complex with Cdc42-GTP, the Pak1 kinase effector and Scd2 [7,8], which leads in vivo to the positive feedback activation of other Cdc42 molecules, as shown in our recent work using optogenetic strategies [6]. The second GEF, Gef1, which localizes to cell poles only in some conditions, promotes Cdc42 activation in response to stress and becomes essential only in absence of Scd1 [9-12]. Three GAPs, namely Rga4, 
Rga6, and Rga3, enhance the intrinsic GTP hydrolytic activity of Cdc42 [13-15]. Rga4 and Rga6 GAPs localize at cell sides, where growth does not occur in non-stressed cells, whereas Rga3 localizes at sites of active growth (cell poles). Fission yeast cells also express a GDI, called Rdi1, though Cdc42 localization and dynamics are not strongly perturbed in its absence $[4,16]$.

Recently, optogenetic studies revealed a novel mechanism that triggers the activation of small GTPases in mammalian cells: Human Rac1 and RhoA, which belong to the same Rho GTPase family as Cdc42, were shown to become active at the cell cortex upon light-dependent cytosolic clustering [17]. In these experiments, the small GTPases were fused to CRY2PHR, the photolyase homology region of A. thaliana cryptochrome 2, which oligomerizes upon blue light exposure. Artificially clustered RhoA induced RhoA signalling-dependent cytoskeletal re-organization and membrane retraction in human cells, suggesting that oligomerization promotes RhoA activation [17]. Ras and Ras-like GTPases are well known to form nanoclusters and dimers at the membrane to activate signal transduction [18-20]. Several Rho-family GTPases, including RhoA, Rac1, Rac2 and Cdc42, were also shown to form dimers or oligomers through homophilic interactions of their polybasic region adjacent to the C-terminal CAAX motif [21,22]. While oligomerization of GTP-bound Cdc42 and Rac1 increases their GTPase activity in vitro, the physiological relevance of clustering of these small GTPases remains to be investigated [22]. In vivo, Rac1-GTP oligomers have been shown to contain several dozen Rac1 molecules together with charged phospholipids and appear to promote signal transduction [21-24]. Cdc42 also forms nanoclusters in Saccharomyces cerevisiae cells $[25,26]$. These nanoclusters show an anisotropic distribution: they accumulate and exhibit larger sizes at cortical sites of polarized growth, in a manner dependent on the scaffold protein Bem1 and anionic membrane lipids [25,27]. Because Bem1 also acts as scaffold that bridges Cdc42-GTP to its GEF and promotes positive feedback activation of Cdc42, Cdc42 nanoclusters may promote Cdc42 feedback activation, though this has not been tested.

In this work, we used an artificial optogenetic strategy to induce the recruitment and clustering of Cdc42 at the plasma membrane of fission yeast cells. We built on our recent work that used the CRY2-CIB1 optogenetic system to probe the positive feedback of Cdc42 [6]. The CRY2-CIB1 system relies on the blue light-induced binding of CRY2PHR (simply denoted CRY2 below) to the N-terminal part of CRY2-binding partner CIB1 (CIBN) [28]. Blue light also induces the formation of CRY2 oligomers [17]. We fused CRY2 to a cytosolic variant of Cdc42 (Cdc42 $\left.2^{\Delta \mathrm{CaX}}\right)$ and co-expressed CIBN linked to the membrane-associated RitC anchor. In our earlier study, we showed that cortical recruitment of a GTP-locked, constitutively active Cdc42 variant (CRY2-Cdc42Q61L, $\Delta \mathrm{CaaX}$ ) led to the Scd2-dependent co-recruitment of its GEF Scd1 and accumulation of endogenous Cdc42, demonstrating feedback amplification [6]. Surprisingly, we also found that cortical recruitment of CRY2-Cdc42 ${ }^{\Delta \mathrm{CaaX}}$ (not GTP-locked) also induced the co-recruitment of Scd2, suggesting the activation of CRY2-Cdc42 ${ }^{\triangle \mathrm{CaaX}}$. In this work, we confirm that CRY2-dependent recruitment of $\mathrm{Cdc} 42^{\Delta \mathrm{CaaX}}$ at lateral sites, where $\mathrm{Cdc} 42$ is normally inactive, promotes its activation. We show that activated clustered Cdc42 is able to recruit its GEF Scd1 through the scaffold Scd2, suggesting that positive feedback is initiated. However, the activation is efficiently countered by Rga4 GAP-mediated Cdc42 inactivation, and does not lead to cell shape alteration, showing the robustness of the cell polarization system.

\section{Results and Discussion}

\subsection{Weak Activation of CRY2-Cdc42 at the Cell Cortex}

To better characterize CRY2-Cdc42 ${ }^{\Delta \mathrm{CaaX}}$, we first measured its kinetics of recruitment to CIBN-RitC at the plasma membrane. Similar to rates measured for CRY2, CRY2-Cdc42 ${ }^{\triangle \mathrm{CaaX}}$ showed a half-time of protein recruitment to the cortex $<1 \mathrm{~s}$ and independent of the length of the blue light $(488 \mathrm{~nm})$ pulses (30 GFP pulses of $50 \mathrm{~ms}=0.92 \mathrm{~s} \pm 0.24 \mathrm{~s} ; 22$ GFP pulses of $250 \mathrm{~ms}=0.98 \mathrm{~s} \pm 0.25 \mathrm{~s} ; 17$ GFP pulses of $500 \mathrm{~ms}=0.99 \mathrm{~s} \pm 0.31 \mathrm{~s}$; Figure S1A,B). CRY2-Cdc42 ${ }^{\Delta \mathrm{CaaX}}$ cells did not exhibit any morphological defects and grew in a bipolar fashion in the dark (Figure S1C). In blue-light, CRY2-Cdc42 ${ }^{\Delta \text { CaaX }}$ cells maintained their characteristic rod-shape and continued growing from the cell tips (Supplementary 
Figure S1D, green cells), while cells with GTP-locked CRY2-Cdc42Q61L, $\triangle \mathrm{CaaX}$ rounded up indicating isotropic growth (Supplementary Figure S1D, blue cells; [6]). These evidences initially suggested that the recruitment of CRY2-Cdc42 $2^{\Delta \mathrm{CaX}}$ to the cell cortex was innocuous and unable to bias the endogenous $\mathrm{Cdc} 42$ and its regulatory network.

We monitored the distribution of Cdc42-GTP using three GFP-tagged markers that specifically associate with Cdc42-GTP: the scaffold Scd2 [7,29], the CRIB bioreporter (Cdc42-Rac1-interactive-binding domain, [30]), and the Cdc42 effector Pak1, which also contains a CRIB domain. These proteins and probe are normally only detected at the poles and division sites of yeast cells, as well as weakly in the nucleus for the first two. As previously described [6], upon blue light-dependent recruitment of CRY2-Cdc42 ${ }^{\Delta \mathrm{CaaX}}$ to the plasma membrane, Scd2-GFP formed stable foci at the cell sides, which increased progressively in intensity and coincided with CRY2-Cdc42 ${ }^{\Delta \mathrm{CaaX}}$ clusters, while Scd2-GFP intensity decreased at cell poles (Figure 1A-D and Figure S2). We had previously shown that Scd 2 was strongly recruited by GTP-locked CRY2- Cdc42 $611 \mathrm{~L}, \triangle \mathrm{CaaX}$ but not GDP-locked CRY2- Cdc42 $2^{\mathrm{T} 17 \mathrm{~N}, \Delta \mathrm{CaaX}}$ [6]. Indeed, we confirmed that CRY2-Cdc42 ${ }^{\triangle \mathrm{CaaX}-\mathrm{T} 17 \mathrm{~N}}$ does not lead to Scd2 foci at cell sides, indicating that CRY2- Cdc42 ${ }^{\Delta \mathrm{CaaX}}$ must be in the GTP-bound form to recruit Scd2 (Figure 1A). CRIB-3GFP also formed dim foci at the cell sides, which became visible 40-60s after light stimulation (Figure 1A,E and Figure S3). The CRIB-3GFP side signal was however weaker and delayed relative to that observed upon light-induced recruitment of GTP-locked $\mathrm{CRY2}-\mathrm{Cdc} 42^{\mathrm{Q} 61 \mathrm{~L}, \Delta \mathrm{CaaX}}$ (Figure 1E; [6]). Indeed, recruitment of CRIB-3GFP was only statistically significant in the second half of the $87 \mathrm{~s}$-time-lapse (see materials and methods). This side recruitment was also mirrored by a reduction of the CRIB signal at the cell poles (Figure 1F). The Pak1-sfGFP traces also showed an upward trajectory on cell sides and a downward trajectory at cell poles but were not statistically different from negative control after $87 \mathrm{~s}$ (Figure 1A,G,H). We note, however, that the higher levels of both CRIB and Pak1 on cell sides were marginally statistically significant after 30 min illumination ( $p=0.04$; see Figure $5 B, C)$. Based on the recruitment of Scd 2 by CRY2-Cdc $42^{\triangle \mathrm{CaaX}}$ on cell sides, these data suggest that the heterologous Cdc42 moiety within the CRY2-Cdc $42^{\triangle \mathrm{CaaX}}$ system is transiently activated when recruited in clusters at the cell sides and sufficient to alter the endogenous sites of Cdc42 activity at cell poles. We hypothesize that the stronger Scd2 than CRIB and Pak1 signals reflect a more stable binding, likely stabilized by additional association, for instance, to anionic lipids [27].

As an alternative strategy to increase Cdc42 levels at the plasma membrane, we overexpressed Cdc42. In this experiment, we used the functional, internally tagged $c d c 42-m C h e r r y{ }^{S W}$ allele [4] expressed under the $p^{a c t 1}$ promoter in cells lacking the endogenous gene, which allowed us to quantify the global increase in expression levels at 3.3-fold (Figure S4A,B). The Cdc42 level increase was roughly uniform around the cell cortex (not shown). Cdc42 overexpression also led to a 1.2-fold increase in the expression of the CRIB-3GFP reporter (expressed under the $p^{\text {pak1 }}$ promoter, Figure S4B). Cdc42 overexpression led to a small increase in CRIB signal at cell poles (even after correction by the 1.2-fold increase in probe expression) and a small increase in cell length ( $p^{a c t 1}-c d c 42$ cell length $=14.2 \pm 1$ $\mu \mathrm{m}$ vs. WT cell length $=13.4 \pm 1.1 \mu \mathrm{m}$, t-test $p$-value $=2.8 \times 10^{-5} ; \mathrm{p}^{\text {act1 }}$-cdc42 cell width $=3.8 \pm 0.3$ $\mu \mathrm{m}$ vs. WT cell width $=3.8 \pm 0.3 \mu \mathrm{m}, t$-test $p$-value $=0.96$ ), suggesting increased Cdc42 activity at cell poles. However, Cdc42 overexpression had no effect on CRIB-3GFP levels at cell sides (Figure $\mathrm{S} 4 \mathrm{C}, \mathrm{D})$. We conclude that activation of CRY2-Cdc42 ${ }^{\triangle \mathrm{CaaX}}$ on cell sides is not simply a consequence of overexpression but may be due to other changes imposed by CRY2 activation. It is possible that CRY2-dependent clustering of Cdc42 directly causes the activation of the GTPase, as has been proposed for other GTPases [17], though unknown mechanism. Alternatively, clustering may have indirect effects, such as slowing down Cdc42 dynamics, which influence its activation cycle. 
A
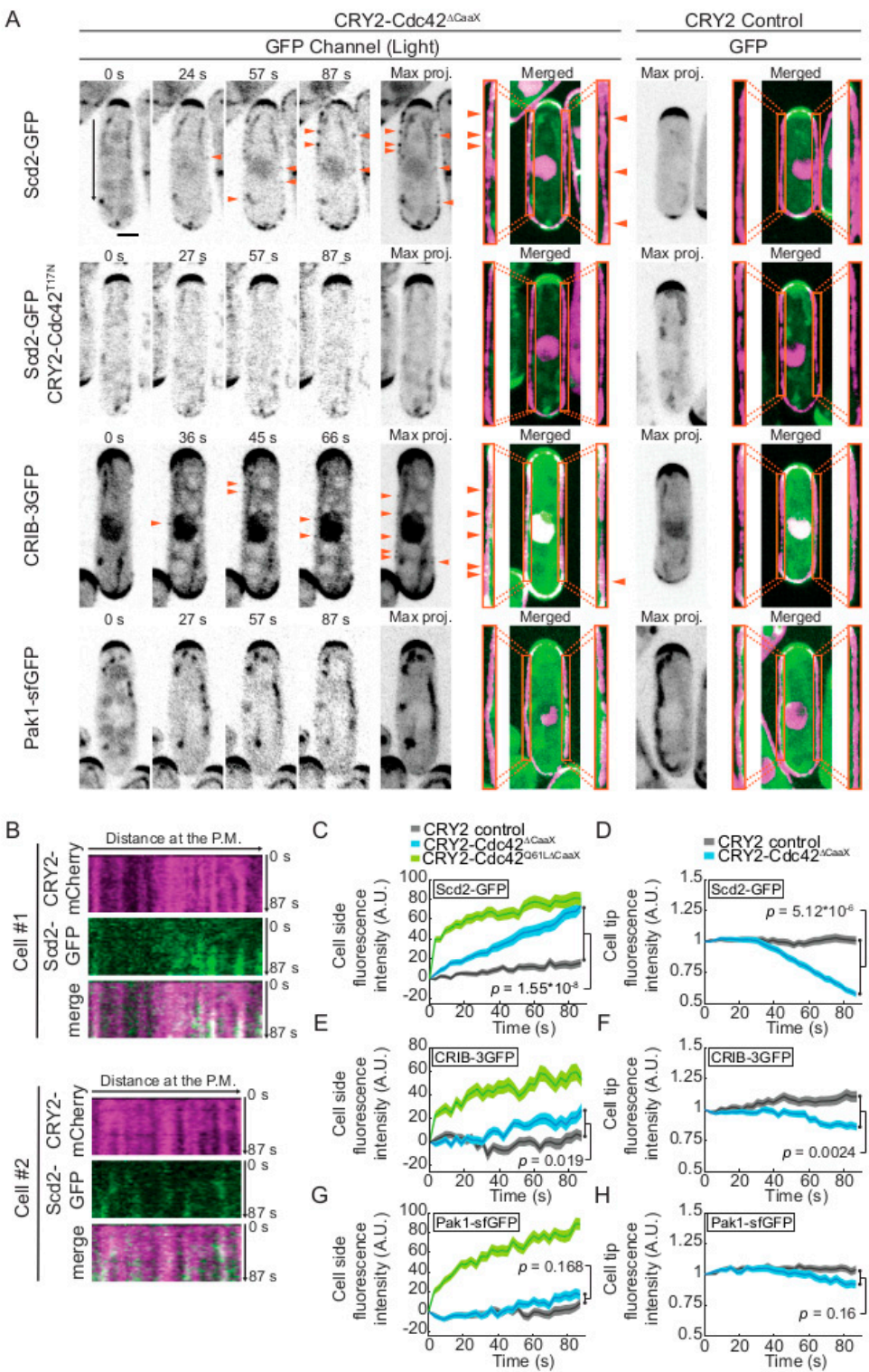

Figure 1. Ectopic sites of Cdc42 activation upon CRY2-Cdc42 ${ }^{\Delta \mathrm{CaaX}}$ cell-sides recruitment. (A) Localization of Scd2-GFP, CRIB-3GFP and Pak1-sfGFP in CRY2-Cdc42 ${ }^{\Delta \mathrm{CaaX}}$-expressing cells (B/W inverted images and green channel in merge). The GFP max projection ("max proj.") images show GFP maximum-intensity projections of 30 time points over $87 \mathrm{~s}$. Merged images are composites of GFP and RFP max projections ( $\mathrm{t} 0$ omitted from the RFP projection). Magnification of the lateral cortex is shown in the orange insets. Arrowheads point to lateral Scd2-GFP and CRIB-3GFP signal. The black arrow in Scd2-GFP panel indicates the cortical region in the kymograph shown in (B, cell \#1). Note that autofluorescent organelles appear as linear and circular structures in some of the GFP channel images. (B) Kymograph at the lateral cell cortex for two cells over the $87 \mathrm{~s}$ (cell \#1 corresponds to the cell shown in (A)). (C,D) Quantification of Scd2-GFP signal intensity at cell sides (C) and cell poles (D). (E,F) Quantification of CRIB-3GFP signal intensity at cell sides (E) and cell poles (F). (G,H) Quantification of Pak1-sfGFP signal intensity at cell sides (G) and cell poles $(\mathbf{H})$. In $(\mathbf{C}-\mathbf{H}), n>66$ cells. Exact numbers are listed in the methods. In $(\mathbf{C}, \mathrm{E}, \mathrm{G})$, the $\mathrm{CRY} 2-\mathrm{Cdc} 42^{\mathrm{Q} 61 \mathrm{~L}, \Delta \mathrm{CaaX}}$ average trace is shown for cell-side recruitment comparison (data from (Lamas et al., 2020)). In all graphs, thick line = average; shaded area $=$ standard error of the mean (SEM); WT, wild type; A.U., arbitrary units. Bars $=2 \mu \mathrm{m}$. Associated trace analysis is shown in Figure S2A for cell-side and Figure S2B for cell pole analyses. 


\subsection{CRY2-Cdc42 Activation in Absence of Cdc42 GEFs}

To probe the mode of CRY2-Cdc42 ${ }^{\triangle \mathrm{CaaX}}$ activation, we repeated the optogenetic experiments above in strains lacking the Cdc42 GEF Scd1. In scd1 $\Delta$ cells, Pak1-sfGFP was not detected at cell sides, similar as in wildtype cells. We also observed only rare CRIB-3GFP dots, and no significant increase in CRIB levels at the sides nor decrease at cell poles of $s c d 1 \Delta$ cells (Figure 2A-C), suggesting that $\mathrm{Scd} 1$ participates in CRY2-Cdc42 ${ }^{\Delta \mathrm{CaOX}}$ activation. However, Scd2-GFP was still recruited to cell sides and decreased from cell poles (Figure 2A-C). Because Scd2 recruitment is strictly dependent on the GTP-bound form of Cdc42 (see Figure $1 A ;[6,7,29]$ ), this suggests that CRY2-Cdc42 ${ }^{\triangle \mathrm{CaaX}}$ may still be active in these cells. We thus probed the role of the second Cdc42 GEF Gef1. In gef1 $\Delta$ cells, Scd2-GFP accumulation at cell sides and decrease at cell poles exhibited similar dynamics as in WT cells (Figure 2D-F). It is possible that the two GEFs work redundantly in this situation, a hypothesis difficult to test due to the lethality of $s c d 1 \Delta$ gef1 $\Delta$ double mutants [11,12]. An alternative hypothesis, which we do not favour, is that Cdc42 clustering through CRY2 binding may promote Scd 2 recruitment independently of its activation.

\subsection{CRY2-Cdc42 Promotes Recruitment of Its GEF Scd1 in Scd2 Scaffold-Dependent Manner}

Because Cdc42-GTP promotes the recruitment of its GEF Scd1 for feedback amplification of Cdc42 activation [6], we probed whether CRY2-Cdc42 ${ }^{\triangle \mathrm{CaaX}}$ induces Scd1 recruitment. Indeed, Scd1 formed weak foci at cell sides upon blue-light activation (Figure 3A,B), similar to the CRIB-3GFP foci observed in CRY2-Cdc42 ${ }^{\Delta \mathrm{CaaX}}$ cells (see Figure $1 \mathrm{~A}$ and Figure S3). The appearance of Scd1 foci at cell sides was also mirrored by a decrease of Scd1-3GFP at the cell tips (Figure 3C). Scd1 recruitment was dependent on the scaffold Scd2, as no cell side accumulation of Scd1-3GFP, nor decrease at cell tips, was detected in $s c d 2 \Delta$ cells (Figure 3D-F). These data suggest that the activated CRY2-Cdc42 ${ }^{\Delta \mathrm{CaaX}}$ is poised to trigger the positive feedback leading to recruitment of its GEF Scd1.

Although $s c d 2$ deletion abolished Scd1 recruitment, it did not substantially affect the accumulation of the CRIB probe (Figure 3D,G), indicating that CRY2-Cdc42 $2^{\Delta \mathrm{CaOX}}$ is still activated in these cells. This observation is in agreement with the finding that CRY2-Cdc42 ${ }^{\Delta \mathrm{CaaX}}$ may be activated independently of Scd1. Because CRIB intensity on cell sides was not reduced in $s c d 2 \Delta$ cells (Figure $3 \mathrm{H}$ ), we conclude that the scaffold-dependent recruitment of the GEF by CRY2-Cdc42 ${ }^{\Delta C a a X}$ does not play a major role in amplifying Cdc42 activation at cell sides.

\subsection{The Cdc42 GAP Rga4 Prevents Isotropic Growth of CRY2-Cdc42 ${ }^{\Delta \mathrm{CaaX}}$ Cells}

If CRY2-Cdc42 ${ }^{\triangle \mathrm{CaaX}}$ is activated at cell sides and recruits its own GEF, why is Cdc42 activity not further amplified by the positive feedback mechanism and does not lead to cell shape changes? Indeed, even long-term growth of CRY2-Cdc42 cells in the light did not change their cell length and width, or aspect ratio, similar to control CRY2 cells. By contrast, constitutive cortical localization of CRY2-Cdc42 $261 \mathrm{~L}, \Delta \mathrm{CaaX}$ by growth in light conditions led to a significant increase in cell width and decrease in cell length, yielding a reduced aspect ratio (Figure $4 \mathrm{~A}-\mathrm{C}$ ). 

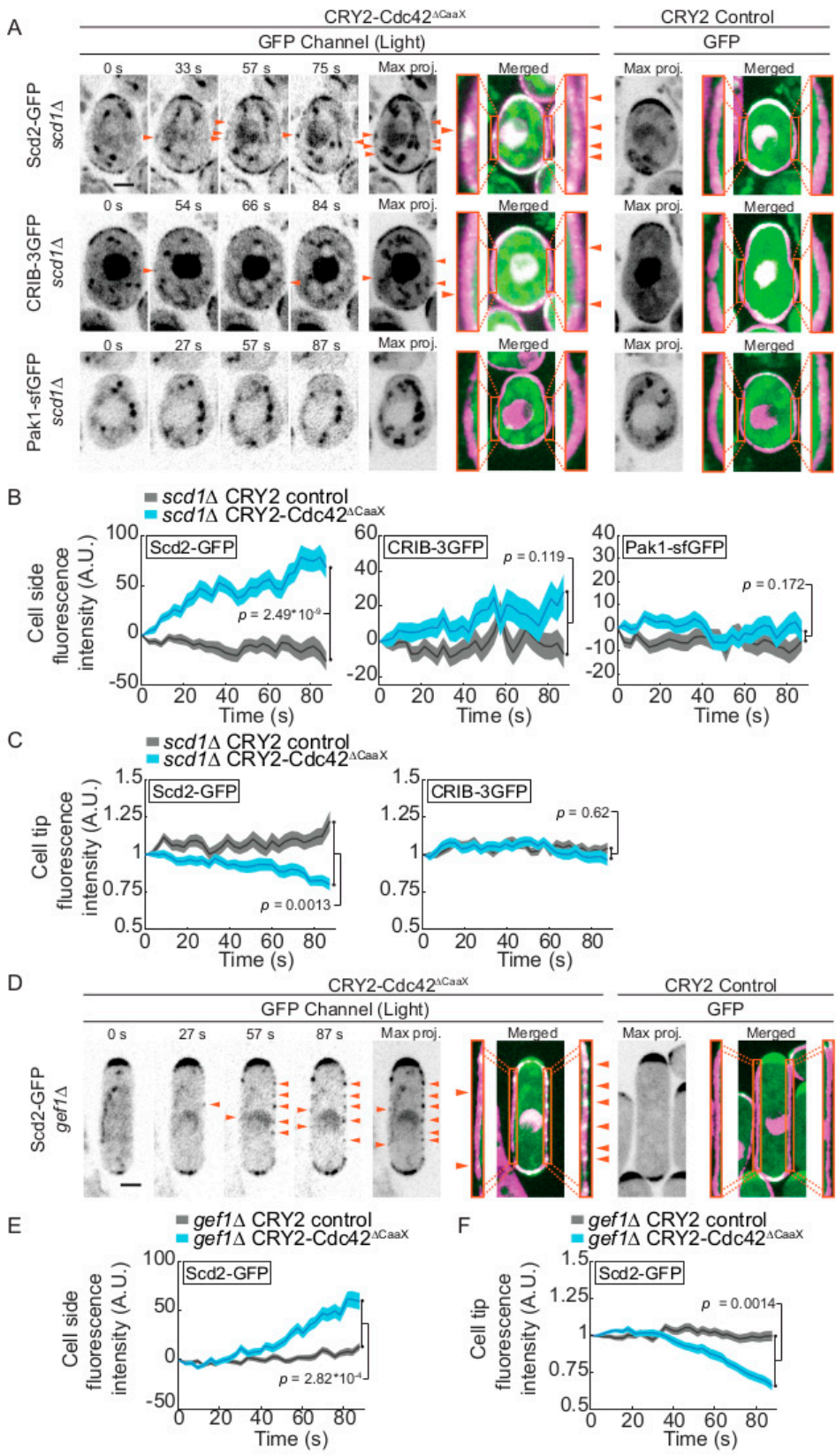

Figure 2. Role of Cdc42 GEFs in activation of CRY2-Cdc42 ${ }^{\triangle \mathrm{CaaX}}$. (A) Localization of Scd2-GFP, CRIB-3GFP and Pak1-sfGFP in CRY2-Cdc42 ${ }^{\Delta \mathrm{CaaX}}$-expressing scd1 $\Delta$ cells (B/W inverted images and green channel in merge). The GFP max projection ("max proj.") images show GFP maximum-intensity projections of 30 time points over $87 \mathrm{~s}$. Merged images are composites of GFP and RFP max projections ( $\mathrm{t} 0$ omitted from the RFP projection). Magnification of the lateral cortex is shown in the orange insets. Arrowheads point to lateral Scd2-GFP and CRIB-3GFP signal. (B) Quantification of GFP signal intensities of Scd2-GFP, CRIB-3GFP and Pak1-sfGFP at cell sides of $s c d 1 \Delta$ mutants. $n>66$ cells. Exact numbers are listed in the methods. (C) Quantification of Scd2-GFP and CRIB-3GFP signal intensity at cell poles of $s c d 1 \Delta$ mutants. $n>62$ cells. Exact numbers are listed in the methods. (D) Localization of Scd2-GFP in CRY2-Cdc42 ${ }^{\Delta \mathrm{CaaX}}$-expressing gef1 $\Delta$ cells. Layout as in panel A. (E,F) Quantification of Scd2-GFP signal intensity at cell sides (E) and cell poles (F) of gef1 $\Delta$ mutants. $n>68$ cells. Exact numbers are listed in the methods. In all graphs, thick line $=$ average; shaded area $=$ standard error of the mean (SEM); WT, wild type; A.U., arbitrary units. Bars $=2 \mu \mathrm{m}$. Associated trace analysis is shown in Figure S5. Autofluorescent organelles appear as linear and circular structures in some of the GFP channel images. 
A

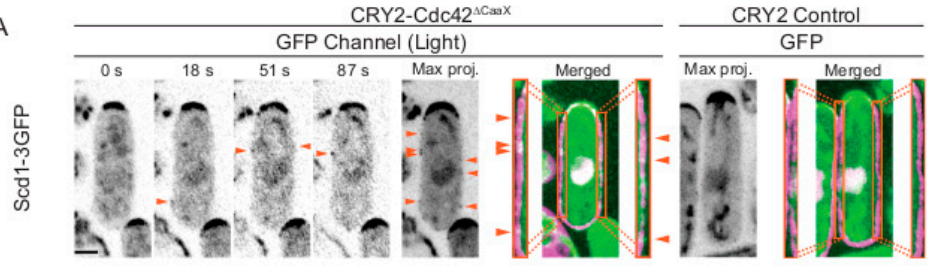

B

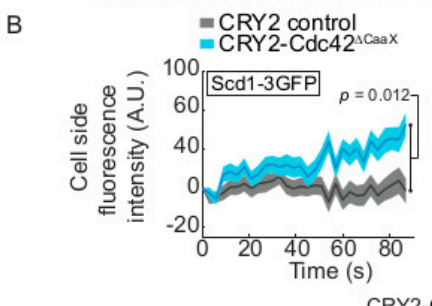

C $\quad$ CRY2 control $=$ CRY2-Cdc42

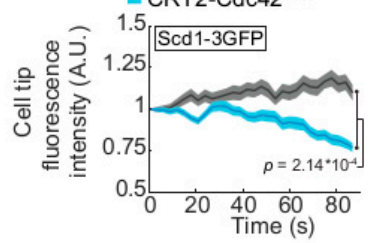

D

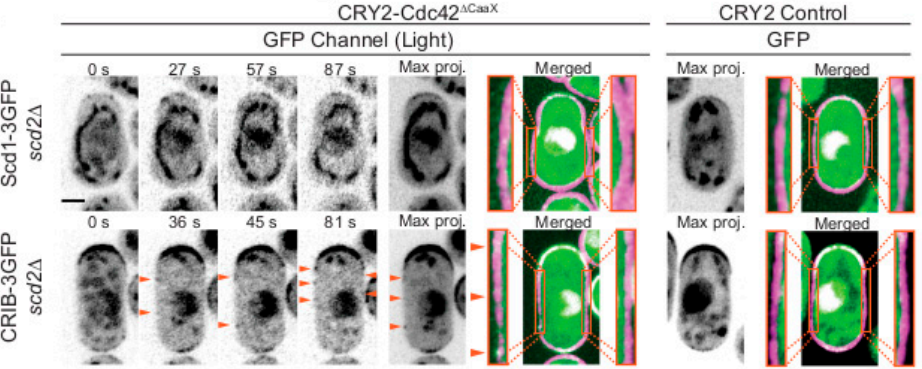

E

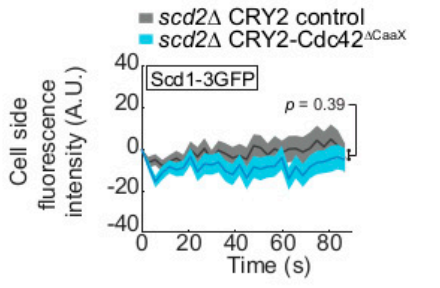

$\mathrm{F}$

= scd2 $2 \Delta$ CRY2 control

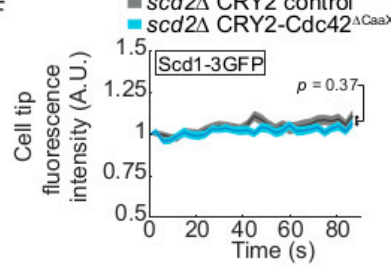

G
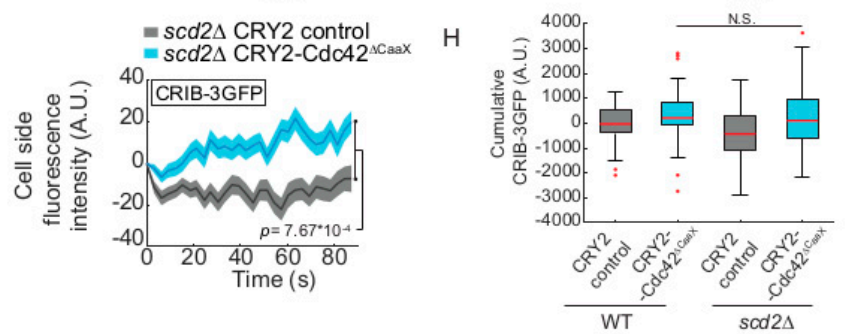

Figure 3. CRY2-Cdc42 ${ }^{\Delta \mathrm{CaaX}}$ recruits the GEF Scd1 dependent on the scaffold Scd2. (A) Localization of Scd1-3GFP in CRY2-Cdc42 ${ }^{\triangle \mathrm{CaaX}}$-expressing cells (B/W inverted images and green channel in merge). The GFP max projection ("max proj.") images show GFP maximum-intensity projections of 30 time points over $87 \mathrm{~s}$. Merged images are composites of GFP and RFP max projections ( $\mathrm{t} 0$ omitted from the RFP projection). Magnification of the lateral cortex is shown in the orange insets. Arrowheads point to lateral Scd1-3GFP signal. (B,C) Quantification of Scd1-3GFP signal intensity at cell sides (B) and cell poles (C). $n>71$ cells. Exact numbers are listed in the methods. (D) Localization of Scd1-3GFP and CRIB-3GFP in CRY2-Cdc42 ${ }^{\Delta \mathrm{CaaX}}$-expressing $s c d 2 \Delta$ cells. Layout as in panel A. (E,F) Quantification of Scd1-3GFP at cell sides (E) and cell poles (F) of $s c d 2 \Delta$ mutant. $n>74$ cells. Exact numbers are listed in the methods. (G) Quantification of CRIB-3GFP at cell sides of $s c d 2 \Delta$ mutants. $n>84$ cells. Exact numbers are listed in the methods. (H) Comparison of cumulative CRIB-3GFP intensities in WT and $s c d 2 \Delta C R Y 2$ and CRY2-Cdc42 ${ }^{\Delta C a a X}$ cells; in $s c d 2^{+} p^{\text {control }}$ vs. ${ }^{C R Y 2-C d c 42}=0.019$ (data from Figure 1E); in $s c d 2 \Delta p^{\text {control }}$ vs. ${ }^{\text {CRY2-Cdc42 }}=7.67 \times 10^{-4}$ (data from panel $3 \mathrm{G}$ ); $p^{\mathrm{CRY} 2-\mathrm{Cdc} 42 \text { in WT }}$ vs. ${ }^{\text {scd } 2 \Delta}=0.4$. In all graphs, thick line = average; shaded area = standard error of the mean (SEM); WT, wild type; A.U., arbitrary units. Bars $=2 \mu \mathrm{m}$. Associated trace analysis is shown in Figure S6. Autofluorescent organelles appear as linear and circular structures in some of the GFP channel images. 
A

\begin{tabular}{|c|c|c|c|c|c|c|c|c|c|}
\hline \multirow[b]{2}{*}{ Genotype } & \multicolumn{3}{|c|}{ Cell length } & \multicolumn{3}{|c|}{ Cell with } & \multicolumn{3}{|c|}{ Aspect ratio } \\
\hline & $\begin{array}{c}\text { CRY2 } \\
\text { control }\end{array}$ & 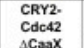 & 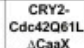 & $\begin{array}{c}\text { CRY2 } \\
\text { control }\end{array}$ & 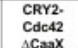 & 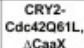 & $\begin{array}{c}\text { CRY2 } \\
\text { control }\end{array}$ & $\begin{array}{l}\text { CRR2. } \\
\text { cdc4t2 }\end{array}$ & 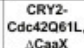 \\
\hline$w T$ & $15.1 \pm 0.09$ & $\begin{array}{l}\frac{A \cos x}{15} \pm 0.04 \\
\end{array}$ & $\frac{1 \operatorname{cosax}}{11.7 \pm 0.07}$ & $3.5 \pm 0.02$ & $\begin{array}{l}\Delta .7 \operatorname{cax} \\
3.7 \pm 0.02\end{array}$ & $\frac{\Delta \operatorname{cosan}}{5.6 \pm 0.04}$ & $4.3 \pm 0.033$ & 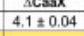 & $\begin{array}{l}\Delta \operatorname{scasx} \\
2.1 \pm 0.02\end{array}$ \\
\hline grass & $13.8 \pm 0.1$ & $13.7 \pm 0.04$ & $11.9 \pm 0.09$ & $4 \pm 0.02$ & $4.4 \div 0.03$ & $5.1 \pm 0.04$ & $3.5 \pm 0.03$ & $3.1 \pm 0.04$ & $24 \pm 0.02$ \\
\hline rgaces & $142 \pm 0.07$ & $13.9 \pm 0.03$ & $10.2 \pm 0.06$ & $4.1 \pm 0.03$ & $4.2 \pm 0.03$ & $5.7 \pm 0.05$ & $3.5 \pm 0.03$ & $3.4 \pm 0.03$ & $1.8 \pm 0.02$ \\
\hline rga3s & $14.1 \pm 0.08$ & $14.1 \pm 0.03$ & $11.5 \pm 0.07$ & $3.5 \pm 0.03$ & $3.8 \pm 0.03$ & $4.7 \pm 0.05$ & $4.0 \pm 0.03$ & $3.8 \pm 0.03$ & $25 \pm 0.02$ \\
\hline rats & $13.5 \pm 0.09$ & $127 \pm 0.02$ & $10.0 \pm 0.05$ & $3.9 \pm 0.03$ & $3.8 \pm 0.02$ & $5.9 \pm 0.02$ & $3.5 \pm 0.03$ & $3.4 \pm 0.02$ & $1.7 \pm 0.02$ \\
\hline gapts & $13.9 \pm 0.10$ & $13.7 \pm 0.03$ & $9 \pm 0.11$ & $4 \pm 0.02$ & $4 \pm 0.02$ & $7.2 \pm 0.06$ & $3.5 \pm 0.03$ & $3.5 \pm 0.03$ & $1.3 \pm 0.02$ \\
\hline rgadsrgas & $11 \pm 0.09$ & $11.1 \pm 0.02$ & $11 \pm 0.07$ & $4.4 \pm 0.02$ & $4.6 \pm 0.03$ & $4.8 \pm 0.03$ & $2.7 \pm 0.02$ & $2.4 \pm 0.02$ & $23 \pm 0.02$ \\
\hline rogatsyoses & $11.4 \pm 0.06$ & $10.1 \pm 0.02$ & $9.6 \pm 0.08$ & $5.2 \pm 0.05$ & $5.7 \pm 0.07$ & $6 \pm 0.05$ & $2.2 \pm 0.02$ & $1.8 \pm 0.02$ & $1.6 \pm 0.02$ \\
\hline rgasasyasen & $13.9 \pm 0.09$ & $13.2 \pm 0.02$ & $10.5 \pm 0.09$ & $4.1 \pm 0.03$ & $4 \pm 0.02$ & $6.3 \pm 0.05$ & $3.4 \pm 0.03$ & $3.3 \pm 0.02$ & $1.7 \pm 0.02$ \\
\hline rodraggats & $12.1 \pm 0.07$ & $11.3 \pm 0.02$ & $9.9 \pm 0.06$ & $4.4 \pm 0.02$ & $5.1 \pm 0.03$ & $6.1 \pm 0.06$ & $2.7 \pm 0.02$ & $2.2 \pm 0.02$ & $1.7 \pm 0.02$ \\
\hline rodiagans & $13.1 \pm 0.07$ & $129 \pm 0.02$ & $9.4 \pm 0.06$ & $4.2 \pm 0.02$ & $4.3 \pm 0.02$ & $6.3 \pm 0.04$ & $3 \pm 0.02$ & $3 \pm 0.02$ & $1.5 \pm 0.01$ \\
\hline redagasa & $13.8 \pm 0.07$ & $13.2 \pm 0.02$ & $10.6 \pm 0.05$ & $4.3 \pm 0.02$ & $4.2 \pm 0.03$ & $5.4 \pm 0.04$ & $3.2 \pm 0.02$ & $3.2 \pm 0.02$ & $2 \pm 0.02$ \\
\hline vgats & . & $122 \pm 0.02$ & 9. & .04 & 6.1 & 07 & $2 \pm 0.02$ & $0 \pm 0.02$ & $3 \pm 0$ \\
\hline & 13.1 & 1.02 & .11 & $4.3 \pm 0.02$ & $4.4 \pm 0.02$ & $8.1 \pm 0.08$ & $1 \pm 0.02$ & $3 \pm 0.02$ & $1.1 \pm 0.01$ \\
\hline prapgasu & $13.5 \pm 0.08$ & $122 \pm 0.02$ & $9 \pm 0.10$ & $4.5 \pm 0.02$ & $3.9 \pm 0.0$ & $7 \pm 0.05$ & $3.3 \pm 0.02$ & $3.4 \pm 0.02$ & $1.4 \pm 0.01$ \\
\hline
\end{tabular}

B
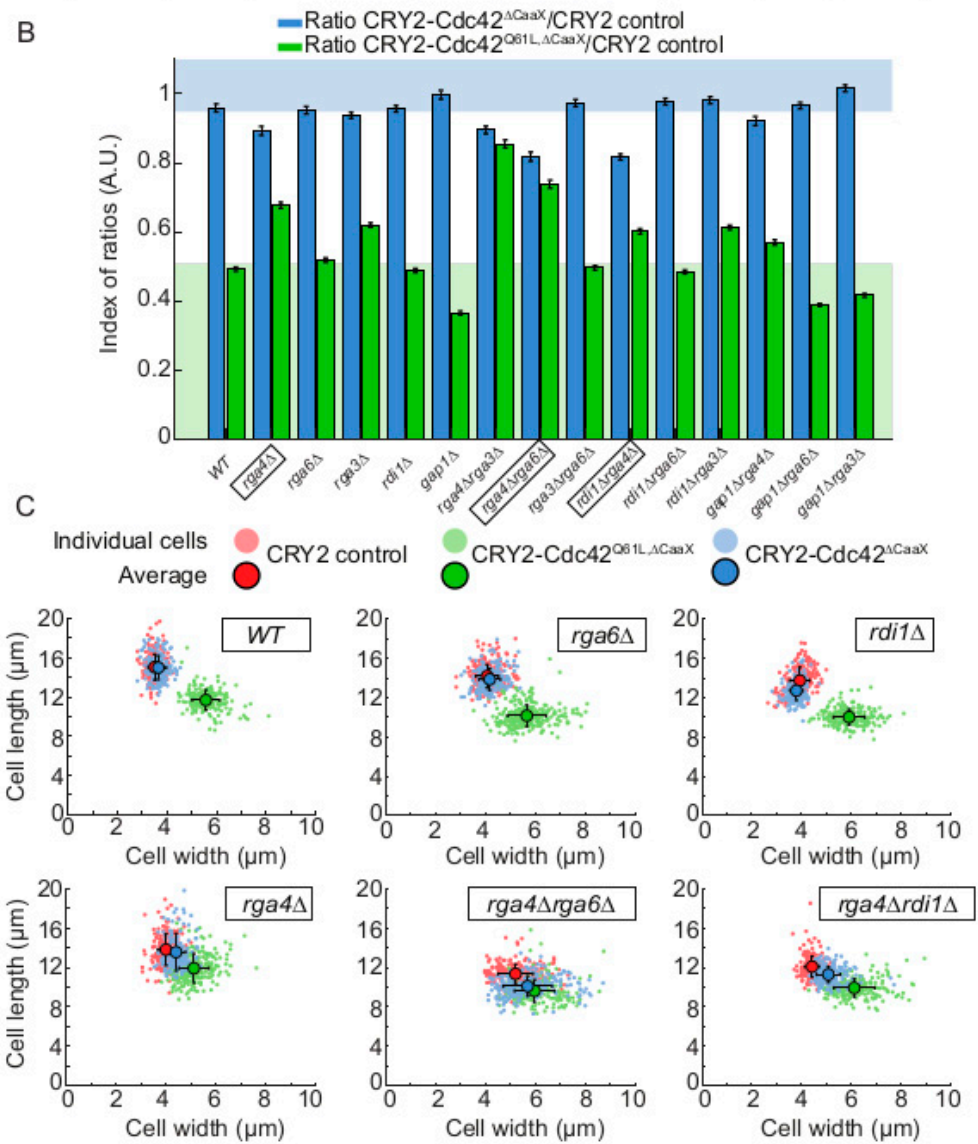

Figure 4. Rga4 GAP prevents growth on cell sides in CRY2-Cdc42 ${ }^{\Delta \mathrm{CaaX}}$ cells. (A) Average cell length $(\mu \mathrm{m})$, cell width $(\mu \mathrm{m})$ and aspect ratio of CRY2, CRY2-Cdc42 ${ }^{\Delta \mathrm{Caax}}$ and CRY2-Cdc42 ${ }^{\mathrm{Q} 61 \mathrm{~L}, \Delta \mathrm{CaaX}}$ mutants. For all mutant $N=2, n>80$ cells per experiment; except for gap1 $\Delta$ mutants, $N=1, n>80$ cells per experiment. (B) Aspect ratios of CRY2-Cdc42 ${ }^{\Delta \mathrm{Caax}}$ and CRY2-Cdc42Q61L, $\Delta$ Caax -expressing cells grown in the light normalized to the aspect ratios of CRY2-expressing cells for all the tested mutants. Bars $=$ standard error. The green background indicates expected reduction in aspect ratio upon CRY2-Cdc42 $261 \mathrm{~L}, \Delta \mathrm{CaaX}$ recruitment in WT cells. The blue background indicates absence of change in aspect ratio upon $\mathrm{CRY} 2-\mathrm{Cdc} 42^{\mathrm{Q} 61 \mathrm{~L}}$ recruitment. Note that all rga4 $\Delta$ mutants fall in the white intermediate space. (C) Cluster plot of length and width in single cells of WT, rga4 $\Delta$, rga6 $\Delta, r g a 4 \Delta r g a 6 \Delta$, rdi1 $\Delta$ and rga4 $\Delta r$ di1 $\Delta$ mutants expressing CRY2, CRY2-Cdc42 ${ }^{\Delta C a a x}$ or CRY2-Cdc42 $261 \mathrm{~L}, \Delta$ CaaX . Small dots = single cells; Large, dark dots = average; bars = standard deviation; A.U., arbitrary units; WT, wild type. 
We hypothesized that the activation of CRY2-Cdc42 4 CaaX at cell sides is rapidly counteracted by negative regulators. We focused our attention on the three Cdc42 GAPs Rga3, Rga4 and Rga6, the GDI protein Rdi1 and the Ras1 GAP Gap1. Rga3, Rga4, and Rga6 directly promote Cdc42-GTP hydrolysis [13-15]. Rdi1 may promote Cdc42 extraction from the membrane, although previous work showed that it is largely dispensable for Cdc42 dynamics in S. pombe $[4,16]$. Gap1 directly promotes Ras1-GTP hydrolysis [31]. As Ras1 promotes Scd1 activation and is uniformly active at the plasma membrane in gap1 $\Delta[6,31,32]$, we hypothesized Scd1 activation on cell sides may be amplified in this mutant. We constructed single and most double deletion mutants expressing either of

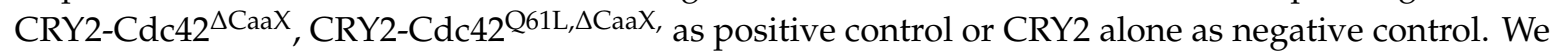
then measured the cell length and cell width of calcofluor-stained dividing cells after at least $14 \mathrm{~h}$ of exponential growth in light conditions and calculated aspect ratios (Figure 4A).

To estimate the change in aspect ratio upon Cdc42 lateral recruitment while taking into account the initial shape of the cell, we normalized the aspect ratios from cells recruiting Cdc42 to those expressing only CRY2 (Figure 4B). CRY2-Cdc42 ${ }^{\mathrm{Q} 61 \mathrm{~L}, \Delta \mathrm{CaaX}}$ led to a $>2$-fold reduction in aspect ratio in WT, rga6 $\Delta$, rdi1 $\Delta$ and gap $1 \Delta$ cells, but had less effect on cell shape change in single and double rga4 $\Delta$ mutants, perhaps in part due to the already wider cell shape of rga $4 \Delta$ cells [14]. Interestingly, CRY2-Cdc $42^{\Delta \mathrm{CaaX}}$ had little effect on aspect ratio in WT or any single mutants, except in rga4 $\Delta$ cells, which became significantly rounder. Similar, more marked effects were also observed in combinations of rga $4 \Delta$ with rga $6 \Delta$ or $r d i 1 \Delta$. The effect of CRY2-Cdc42 $2^{\Delta \mathrm{CaaX}}$ on the shape of these mutants can also readily be observed in plots of cell width to cell length, with the CRY2-Cdc42 $2^{\Delta \mathrm{CaXX}}$ cell population placed at an intermediate position between the negative CRY2 and positive CRY2-Cdc42Q61L, $\Delta$ CaaX controls (Figure 4C). These data indicate that the optogenetic-dependent Cdc42 activation is counteracted by Cdc42 GAPs placed at cell sides.

We hypothesized that Rga4 and Rga6 directly antagonize Cdc42 activity at cell sides. To probe this idea directly, we imaged CRIB-3GFP and Pak1-sfGFP for 87s immediately after CRY2-Cdc42 4 CaaX recruitment. Both of these Cdc42-GTP interactors showed significantly increased levels at the sides of rga4 $\operatorname{rga} a \Delta$ cells (Figure 5A). The data for Pak1 contrast with what we observed in wildtype cells, where no significant increase was detected at this timepoint (see Figure 1G). To strengthen these data further, we probed for CRIB-3GFP and Pak1-sfGFP localization at cell sides at a later timepoint, after $30 \mathrm{~min}$ of continuous white-light illumination, similar to the light conditions used to assess cell shape changes. Compared to the small increase of CRIB-3GFP and Pak1-sfGFP at the sides of wildtype cells in these conditions, rga4 $\operatorname{rga} 6 \Delta$ mutants showed higher increase, which was highly statistically significant (Figure 5B,C). These data support the view that Cdc42 GAPs at cell sides antagonize the CRY2-Cdc42 ${ }^{\triangle \mathrm{CaaX}}$ activation to prevent cell widening. A second, non-mutually exclusive possibility is that the cell shape change observed in rga4 $\Delta$ cells reflects the weakening of polarity at the native sites at cell poles, as shown by the decrease of Scd2 and CRIB at cell poles (see Figure 1D,F). Thus, both local and competition effects with native polarity sites may combine to alter the shape of CRY2-Cdc42 $2^{\Delta \text { CaaX }}$-expressing rga $4 \Delta$ cells. 


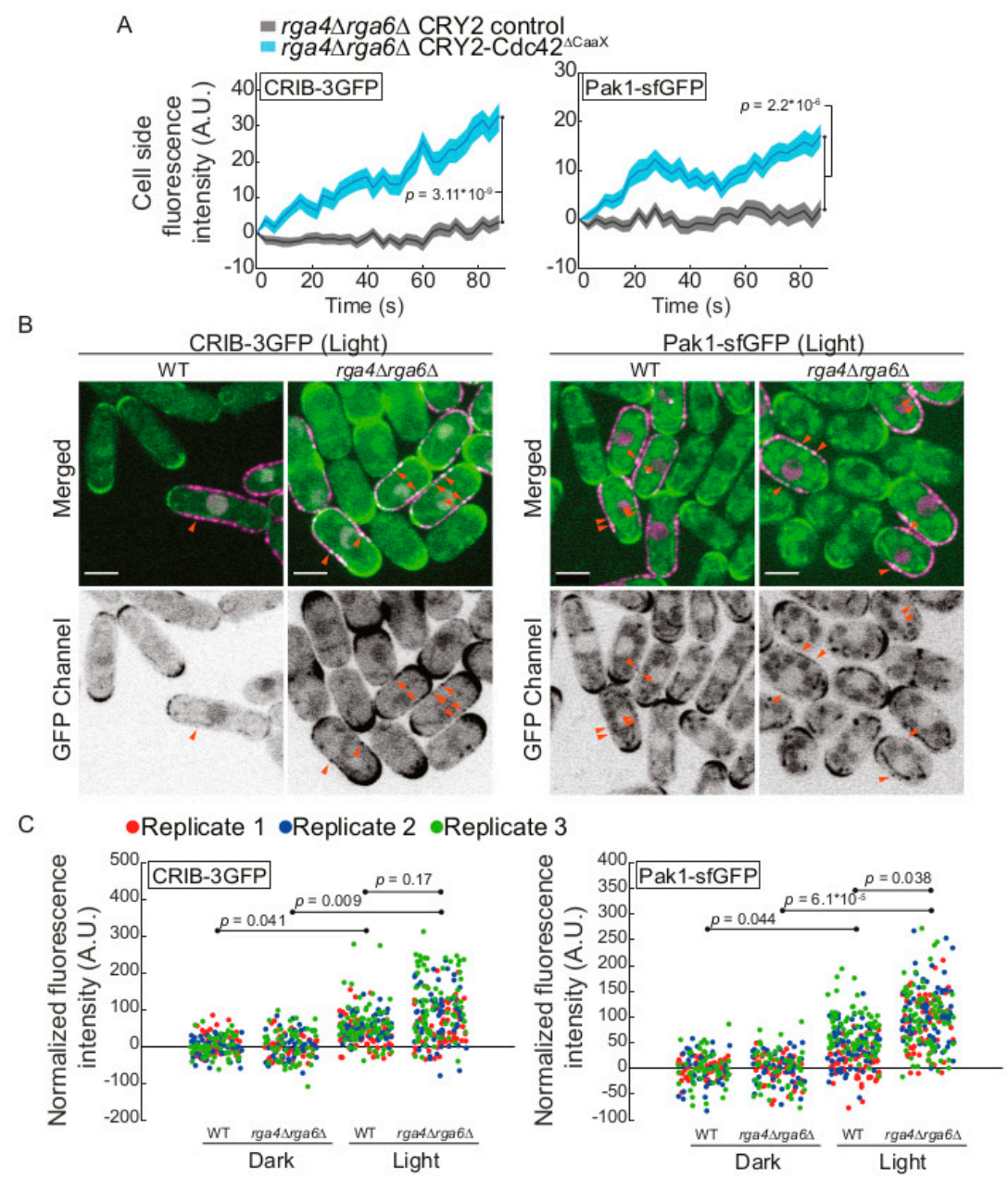

Figure 5. Stronger CRY2-Cdc42 ${ }^{\Delta C a a X}$ activation in cells lacking lateral Cdc42 GAPs. (A) Quantification of CRIB-3GFP and Pak1-sfGFP signal intensity at cell sides of rga4 $\Delta$ rga $6 \Delta$ mutants, $n>80$ cells. Exact numbers are listed in the methods. (B) Localization of CRIB-3GFP and Pak1-sfGFP in control and CRY2-Cdc42 ${ }^{\Delta \mathrm{CaaX}}$-expressing cells upon 30 min-exposure to light (B/W inverted images and green channel in merge). Merged images are composites of GFP and RFP single middle-section images. Arrowheads point to lateral CRIB-3GFP and Pak1-sfGFP signal. Control cells lack the CRY2-Cdc42 ${ }^{\Delta C a a X}$ magenta signal in the merged images. (C) Cluster plots of CRIB-3GFP and Pak1-sfGFP signal intensity at cell sides of WT and rga4 $\Delta$ rga $6 \Delta$ mutants, $n>138$ cells. Exact numbers are listed in the methods. The three experimental replicates are plotted with different colours. Statistical $p$-values are from t-test across experimental replicates $(n=3)$. In all graphs, thick line = average; shaded area $=$ standard error of the mean (SEM); WT, wild type; A.U., arbitrary units. Bars $=4 \mu \mathrm{m}$. Associated trace analysis is shown in Figure S8. Autofluorescent organelles appear as linear and circular structures in some of the GFP channel images.

In summary, the data presented in this work show that CRY2-clustered Cdc42 is ectopically activated at cell sides and leads to an alteration of the native polarity sites at cell poles. It is possible that CRY2-tagging interferes with Cdc42 GTPase activity, such that CRY2-Cdc42 ${ }^{\Delta C a a X}$ represents a slightly activated Cdc42 variant. Alternatively, the CRY2-dependent clustering may lead to Cdc42 activation, as proposed for other GTPases, such as Rac1 and RhoA in mammalian cells [17]. CRY2-clustered Cdc42 leads to very clear Scd2 recruitment, but much weaker CRIB and Pak1 recruitment, raising the question of why these Cdc42-GTP binding proteins behave differently. We hypothesize that this is explained by the stabilization of Scd2 localization through additional interactions for instance with membrane lipids. Interestingly, the activity level of CRY2-Cdc $42^{\Delta \mathrm{CaaX}}$ at cell sides is sufficient to recruit 
the GEF Scd1, whose recruitment to Cdc42-GTP depends on the scaffold Scd2 [6]. However, positive feedback does not appear to become established as Cdc42 activity levels at cell sides are not altered in absence of Scd2. It is possible that the non-physiological linkage of Cdc42 to the plasma membrane (through RitC-CIBN-CRY2 binding instead of through the normal prenyl group) undermines the positive feedback, although we previously showed that a similarly engineered constitutively active Cdc42 construct did trigger feedback-dependent recruitment of endogenous Cdc42 [6]. We favour the view that cellular regions may not be equally permissive to Cdc42 activity feedback amplification. For instance, the absence of Ras1 activity on cell sides may reduce the effectiveness of the feedback [6]. Our data further indicate that the inefficient positive feedback at cell sides may be due to the action of the cell side-localized Cdc42 GAP Rga4, which we have shown antagonizes the effect of CRY2-Cdc42 ${ }^{\triangle \mathrm{CaaX}}$ on effector recruitment and cell morphogenesis. Additional mechanisms preventing growth on cell sides are also being proposed [33]. Thus, one critical question for future research is to better understand the many layers that confer robustness to cell morphogenesis.

\section{Materials and Methods}

\subsection{Strains, Media, and Growth Conditions}

Strains used in this study are listed in Supplementary Table S1. Standard genetic manipulation methods for $S$. pombe transformation and tetrad dissection were used to generate the strains listed. For microscopy experiments, cells were first pre-cultured in $3 \mathrm{~mL}$ of Edinburgh minimal media $(E M M)$ in dark conditions at $30{ }^{\circ} \mathrm{C}$ for $6-8 \mathrm{~h}$. Once exponentially growing, pre-cultures were diluted (Optical Density (O.D.) $600 \mathrm{~nm}=0.02$ ) in $10 \mathrm{~mL}$ of EMM and incubated in dark conditions overnight at $30^{\circ} \mathrm{C}$. In order to allow proper aeration of the culture, $50 \mathrm{~mL}$ Erlenmeyer flasks were used. For cell size analyses cells were pre-cultured and diluted once in $3 \mathrm{~mL}$ of Edinburgh minimal media (EMM) in dark conditions at $30^{\circ} \mathrm{C}$ for $6-8 \mathrm{~h}$. Once exponentially growing, pre-cultures were diluted in $10 \mathrm{~mL}$ of EMM and incubated in light conditions for a minimum of $14 \mathrm{~h}$. All live-cell imaging was performed on EMM-ALU agarose pads, except calcofluor-white experiments in which cells were placed directly on a slide [34]. Gene tagging was performed at endogenous genomic locus at the $3^{\prime}$ end, yielding C-terminally tagged proteins, as described [35]. Pak1 gene tagging was performed by transforming a WT strain with AfeI linearized $\left(\mathrm{pBSII}\left(\mathrm{KS}^{+}\right)\right)$-based single integration vector (pAV72-3'UTR ${ }^{\text {pak1 }}$-Pak1-sfGFP-kanMX-5'UTR ${ }^{\text {pak1 }}$ ) targeting the endogenous locus [36]. The functional mCherry-tagged and sfGFP-tagged $c d c 42$ alleles $c d c 42-m C h e r r y^{s w}$ and $c d c 42$-sfGFP ${ }^{s w}$ were used as described in [4]. Gene tagging, deletion, and plasmid integration were confirmed by diagnostic PCR for both sides of the gene.

The construction of plasmids and strains expressing CIBN-mTagBFP2-Ritc, CRY2, CRY2-Cdc42 ${ }^{\Delta \mathrm{CaaX}}$ and CRY2-Cdc42 $2^{\mathrm{Q} 61 \mathrm{~L}, \Delta \mathrm{CaaX}}$ was done as described in [6].

To generate the Pact1-cdc42-mCh ${ }^{S W}$ strain a pINT-ura $4^{+}$integrative vector was generated. Pact1 was amplified from gDNA using primers osm2378 (atgggccegctagcatgcGATCTACGATAATGAGACGG) and osm2379 (ccggctcgagGGTCTTGTCTTTTGAGGGTT) and cloned using ApaI and Xhol. Cdc42-mCh ${ }^{S W_{-n}} t_{\text {terminator }}$ was amplified from pSM1224 using osm2343 (cccaagcttATGCCCACCATTAAGTGTGTCG) and osm2344 (gctctagaCTTCTAATTACACAAATTCCG) and cloned using HindIII and XbaI. As a results pSM1449 was generated. This plasmid was linearized with AvrII and integrated at ura4 locus of YSM485 strain. The endogenous allele of $c d c 42$ was deleted using a hygromycin $\left(h p h^{+}\right)$resistance cassette as described [35]. $\mathrm{Hph}^{+}$cassette was amplified from pSM693 using osm2511 (TACTTAGGGGTTTGAACTTTCTAGGAATTCAATAAAGTGAAGCAAAGCTTTACGATTAATTAT TTTTTGTGAAATAGTcggatcccegggttaattaa) and osm2512 (AAGCTAAGACATTGTTTACTGTTGTAAA CTAGCTGTATTAAGGAAATTTCGGAAAAGGAAAGAAAACCAGGGGTTAAAgaattcgagctcgtttaaac). Finally, strain YSM3732 was generated by transforming the $C d c 42-m C h^{S W}-n m t_{\text {terminator }}$ strain with the $h p h^{+}$ resistance cassette. 
In primer sequences, restriction sites are underlined. Plasmid maps are available upon request.

\subsection{Cell Length and Width Measurements}

For cell length and width measurements shown in Figure 4 and Supplementary Figure S1, cells were grown at $30^{\circ} \mathrm{C}$ in $10 \mathrm{~mL}$ EMM in light and dark conditions respectively. In this case, we used white light, which we found is sufficient to activate CRY2, shown by the cell rounding triggered by

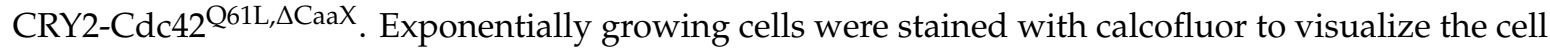
wall and imaged on a Leica epifluorescence microscope with $60 \times$ magnification platform described previously [34]. Measurements were made with ImageJ on septating cells. For each experiment, strains with identical auxotrophies were used.

Aspect ratios were calculated as:

$$
\text { Aspect ratio }=\frac{\text { Cell length }}{\text { Cell width }}
$$

The index of ratios shown in Figure 4B was calculated as:

$$
\begin{gathered}
\text { Index }_{C d c 42 \Delta C a a X}=\frac{\text { Aspect ratio }(C R Y 2 C d c 42 \Delta C a a X)}{\text { Aspect ratio }(C R Y 2)} \\
\text { Index }_{C d c 42 Q 61 L \Delta C a a X}=\frac{\text { Aspect ratio }(C R Y 2 C d c 42 \Delta Q 61 L C a a X)}{\text { Aspect ratio }(C R Y 2)}
\end{gathered}
$$

\subsection{Microscopy}

Fluorescence microscopy experiments were done in a spinning disk confocal microscope, essentially as described [34,37]. Image acquisition was performed on a Leica DMI6000SD inverted microscope equipped with an HCX PL APO 100X/1.46 numerical aperture oil objective and a PerkinElmer Confocal system. This system uses a Yokagawa CSU22 real-time confocal scanning head, solid-state laser lines and a cooled 14-bit frame transfer EMCCD C9100-50 camera (Hamamatsu) and is run by Volocity (PerkinElmer). When imaging strains expressing the CRY2-Cdc42 ${ }^{\triangle \mathrm{CaaX}}$ and/or CRY2 systems, an additional long-pass color filter (550 nm, Thorlabs Inc., Newton, NJ, USA) was used for bright-field (BF) image acquisition, in order to avoid photo-activation by white light.

Spinning disk confocal microscopy experiments shown in Figures 1-3, Supplementary Figures S1A,D, S3, and S7 were carried out using cell mixtures [6]. Cell mixtures were composed by one strain of interest (the sample optogenetic strain, expressing or not an additional GFP-tagged protein) and 2 control strains, namely:

(1) RFP control: An RFP bleaching correction strain, expressing cytosolic CRY2PHR-mCherry.

(2) GFP control: A wild type strain expressing the same GFP-tagged protein as the strain of interest but without the optogenetic system. This strain was used both as negative control for cell side re-localization experiments and as GFP bleaching correction strain (in Figures 1-3 and 5 and related Figures S2, S5, S6 and S8).

Strains were handled in dark conditions throughout. Red LED light was used in the room in order to manipulate strains and to prepare the agarose pads. Strains were cultured separately. Exponentially growing cells (O.D.600nm $=0.4-0.6$ ) were mixed with 2:1:1 (strain of interest, RFP control and GFP control) ratio, and harvested by soft centrifugation ( $2 \mathrm{~min}$ at $1600 \mathrm{rpm})$. The cell mixture slurry (1 $\mu \mathrm{l}$ ) was placed on a $\%$ EMM-ALU agarose pad, covered with a \#1.5-thick coverslip, and sealed with VALAP (vaseline, lanolin, and paraffin). Samples were imaged after 5-10 min of rest in dark conditions.

The plasma membrane recruitment dynamics of CRY2-Cdc42 ${ }^{\Delta \mathrm{CaaX}}$ and CRY2 systems were assessed using cell mixtures. Protein recruitment dynamics were assessed by applying the 3 different photo-activating cycles listed below. Lasers were set to $100 \%$. Shutters were set to maximum speed 
and in all instances the RFP channel was imaged first, before the GFP channel. The duration of the experiment was equal regardless of the exposure time settings $(\approx 15 \mathrm{~s})$ :

(1) $50 \mathrm{~ms}$ : RFP channel $(200 \mathrm{~ms})$, GFP channel $(50 \mathrm{~ms})$. This constitutes one cycle $(\approx 0.5 \mathrm{~s}) .30$ time points were acquired $(\approx 0.5 \mathrm{~s} * 30=15.1 \mathrm{~s})$.

(2) $250 \mathrm{~ms}$ : RFP channel (200 ms), GFP channel ( $250 \mathrm{~ms})$. This constitutes one cycle ( $\approx 0.7 \mathrm{~s}) .22$ time points were acquired $(\approx 0.7 \mathrm{~s} * 22=15.1 \mathrm{~s})$.

(3) 500 ms: RFP channel ( $200 \mathrm{~ms})$, GFP channel $(500 \mathrm{~ms})$. This constitutes one cycle $(\approx 0.9 \mathrm{~s}) .17$ time points were acquired $(0.9 \mathrm{~s} * 17=15.5 \mathrm{~s})$.

Endogenous GFP-tagged protein re-localization experiments were carried out using cell mixtures. Lasers were set to $100 \%$; shutters were set to sample protection and in all instances the RFP channel was imaged first and then the GFP channel. RFP exposure time was always set to $200 \mathrm{~ms}$, whereas the GFP exposure time varied depending on the monitored protein. Cells were monitored in these conditions for $87 \mathrm{~s}$.

Spinning disk confocal time (sum) projections of five consecutive single plane images are shown in Supplementary Figure S4. Z-stack images were acquired on a Spinning disk confocal microscope using an optimal z-spacing of $0.71 \mu \mathrm{m}$ between successive stacks. 6 stacks were acquired (covered $\mathrm{Z}$ distance $=4 \mu \mathrm{m}$ ). In Figure 5, single plane GFP and RFP images were combined to generate the merged (Figure 5B) and used for the GFP signal analyses at the cell side (Figure 5C). Cells were cultured as stated above and placed on EMM-ALU pads. Pads were kept for $30 \mathrm{~min}$ in the dark or under white-light illumination for $30 \mathrm{~min}$ prior imaging.

\subsection{Image Analysis}

All image-processing analyses were performed with Image J software (http://rsb.info.nih.gov/ij/). Image and time-lapse recordings were imported to the software using the Bio-Formats plugin (http://loci.wisc.edu/software/bio-formats). Time-lapse recordings were aligned using the StackReg plugin (http://bigwww.epfl.ch/thevenaz/stackreg/) according to the rigid body method. All optogenetic data analyses were performed using MATLAB (R2019a), with scripts developed in-house. Figures were assembled with Adobe Photoshop CC2019 and Adobe Illustrator 2020.

\subsubsection{CRY2-Cdc42 $2^{\triangle \mathrm{CaaX}}$ and CRY2 Quantifications}

The plasma membrane recruitment dynamics of CRY2-Cdc42 ${ }^{\triangle \mathrm{CaaX}}$ and CRY2 systems was assessed by recording the fluorescence intensity over a 15-pixel long by 36-pixel wide ROI (roughly $1.25 \mu \mathrm{m}$ by $3 \mu \mathrm{m}$ ), drawn perpendicular to the plasma membrane of sample cells, from outside of the cell towards the cytosol. The fluorescence intensity values across the length of the ROI were recorded over time in the RFP channel, in which each pixel represents the average of the width (36 pixels) of the ROI (3 replicates, 30 cells per replicate). Average background signal was measured from tag-free wild-type cells incorporated into the cell mixture. The total fluorescence of the control RFP strain was also measured over time in order to correct for mCherry fluorophore bleaching. In both cases, the ROI encompassed whole cells, where ROI boundaries coincide with the plasma membrane.

Photobleaching correction coefficient was calculated by the following formula:

$$
\text { RFP bleaching correction coef ficient }=\frac{\left(\text { RFP Intensit } y_{t n}-N o G F P B c k g_{t n}\right)}{\left(\text { RFP Intensity } y_{t 0}-N o G F P B c k g_{t 0}\right)}
$$

where RFP intensity is the signal measured from single RFP control cells, NoGFPBckg is the average background signal measured from tag-free cells, $t_{n}$ represents a given time point along the time course of the experiment and $t_{0}$ represents the initial time point $(n=30$ time points). These coefficients were corrected by a moving average smoothing method (moving averaged values $=5$ ). RFP bleaching correction coefficient values calculated for individual RFP control cells were averaged in order to correct for bleaching of the RFP signal. 
The fluorescence intensity values of optogenetic cells were corrected at each time point with the following formula:

$$
\text { RFP intensity }=\left(\left(\text { Raw RFP signal }{ }_{t n}-\text { NoGFPBckg }_{t n}\right) /{\text { RFP bleaching correction coef ficient } \left.t_{t n}\right)}\right.
$$

where Raw RFP signal is for the RFP values measured from sample strains, NoGFPBckg is the average background signal measured from tag-free cells and $t_{n}$ represents a given time point along the time course of the experiment ( $n=30$ time points). The profiles resulting from these analyses were used to get the net plasma membrane recruitment profiles (Figure S1A, [6]), the fluorescence intensities from the peak \pm 1 pixel were averaged and plotted over time.

$$
\text { Peak RFP intensity } y_{t n}=\frac{\left(\text { RFP intensity } \text { peak }-1 \text { pixel tn }_{\text {n }}+\text { RFP intensity }_{\text {peak }}+n+R F P \text { intensity }_{\text {peak }+1 \text { pixel tn }}\right)}{3}
$$

Net P.M. recruitment Profile $=\left(\right.$ Peak RFP intensity $y_{t n}-$ Peak RFP intensity $\left.y_{t 0}\right)$

Finally, the single-cell plasma membrane recruitment half-times were calculated by fitting the normalized recruitment profiles with the following formula:

$$
\begin{gathered}
\text { RFP intensity }(y)=a *\left(1-e^{(-b * t)}\right) \\
\text { Recruitment } t_{1 / 2}=\frac{\ln (0.5)}{b}
\end{gathered}
$$

\subsubsection{Quantifications of the Re-Localization of GFP-Tagged Proteins to Cell Sides}

Endogenous GFP-tagged protein re-localization was assessed upon photo-activation of CRY2-Cdc $42^{\Delta \mathrm{CaaX}}$ and CRY2 systems by recording the fluorescence intensity over a 3 pixel-wide by 36 pixel-long $(\approx 0.25 \mu \mathrm{m}$ by $3 \mu \mathrm{m})$ ROI drawn parallel to the cell side cortex of sample cells. The average fluorescence intensity values of both GFP and RFP channels were recorded over time from sample strains. In these particular experiments, a GFP control strain was included. These strains serve 2 purposes:

(1) Calculation of the GFP bleaching correction coefficient (see below).

(2) Negative control of the experiment: These strains carry the same endogenous GFP-tagged protein as the sample strain of the experiment, however lacking the optogenetic system. This controlled that GFP fluorescence changes were due to the optogenetic system and not caused by imaging per se. Control GFP strains were imaged in the same pad and analysed in the same way as optogenetic cells.

To derive photobleaching correction coefficients, the average camera background signals ( $B c k g$ ) from 5 cell-free regions was measured as above, and fluorophore bleaching from RFP control and GFP control strains were measured at the cell side of control RFP and control GFP strains, for RFP and GFP channels, respectively.

$$
\begin{aligned}
& \text { RFP bleaching correction coefficient }=\frac{\left(\text { RFP Intensit } y_{t n}-B c k g_{t n}\right)}{\left(\text { RFP Intensit } y_{t 0}-B c k g_{t 0}\right)} \\
& \text { GFP bleaching correction coefficient }=\frac{\left(\text { GFP Intensit } y_{t n}-B c k g_{t n}\right)}{\left(G F P \text { Intensit } y_{t 0}-B c k g_{t 0}\right)}
\end{aligned}
$$

where RFP intensity and GFP intensity stand for the signal measured from RFP control and GFP control cells, respectively, $t_{n}$ represents a given time point along the time course of the experiment and $t_{0}$ represents the initial time point ( $n=30$ time points). These coefficients were corrected by a moving average smoothing method, as above. 
The fluorescence intensity values of optogenetic cells in both GFP and RFP channels were independently analysed as follows. First, GFP and RFP signals were background and bleaching corrected, using Formulas (9) and (10) for the RFP and GFP channels, respectively:

$$
\text { P.M. GFP } / \text { RFP } \text { value }_{t n}=\left(\left(\text { Raw signal }_{t n}-\text { Bckg }_{t n}\right) / \text { bleaching correction coefficient } t_{n}\right)
$$

where Raw signal intensity represents the GFP or RFP raw values at the cell side cortex, Bckg stands for the average fluorescence intensity of 5 independent cell-free regions and $t_{n}$ represents a given time point along the time course of the experiment ( $n=30$ time points). The net fluorescence intensity at the cell side cortex was then calculated for both GFP and RFP signals.

Net P.M. GFP $/$ RFP value $_{t n}=\left(\right.$ Fluorescence intensity $_{t n}-$ Fluorescence intensity $\left._{t 0}\right)$

From here on, RFP and GFP signals were treated differently. Single cell plasma membrane RFP profiles from Equation (10) were individually normalized and fitted to the Equation (5) in order to extrapolate the parameter b. Using the Equation (6), recruitment half times of CRY2 and CRY2-Cdc42 ${ }^{\triangle \mathrm{CaaX}}$ were calculated. Because of lower signal-to-noise of the weak GFP fluorescence, plasma membrane GFP profiles from Equation (10) were averaged $(n>20$ profiles per experiment). Measurements from cells obtained in 3 experimental replicates are plotted on Figure $1 \mathrm{C}$ (CRY2-Cdc42 ${ }^{\Delta \mathrm{CaaX}} n=84$ cells; CRY2 control $n=84$ cells, WT control $n=252$ cells); Figure 1E (CRY2-Cdc42 ${ }^{\Delta \mathrm{CaaX}} n=89$ cells, CRY2 control $n=78$ cells, WT control $n=247$ cells); Figure 1G (CRY2-Cdc42 ${ }^{\Delta \mathrm{CaaX}} n=90$ cells, CRY2 control $n=85$ cells, WT control $n=280$ cells); Figure $2 \mathrm{~B}$ (Scd2-GFP scd1 $\Delta$ : CRY2-Cdc42 ${ }^{\Delta \mathrm{CaaX}} n=67$ cells, CRY2 control $n=71$ cells, WT control $n=226$ cells; CRIB-3GFP scd1 $\Delta$ : CRY2-Cdc42 ${ }^{\Delta \mathrm{CaaX}} n=75$ cells, CRY2 control $n=69$ cells, WT control $n=222$ cells; Pak1-sfGFP scd1 $\Delta$ : CRY2-Cdc42 $2^{\Delta \mathrm{CaaX}} n=78$ cells, CRY2 control $n=67$ cells, WT control $n=$ 231 cells), Figure $2 \mathrm{E}\left(\mathrm{CRY} 2-\mathrm{Cdc} 42^{\Delta \mathrm{CaaX}} n=74\right.$ cells, CRY2 control $n=86$ cells, WT control $n=247$ cells); Figure 3B (CRY2-Cdc $42^{\Delta \mathrm{CaaX}} n=79$ cells, CRY2 control $n=72$ cells, WT control $n=228$ cells); Figure 3E (CRY2-Cdc42 ${ }^{\Delta \mathrm{CaaX}} n=81$ cells, CRY2 control $n=75$ cells, WT control $n=257$ cells); Figure 3G (CRY2-Cdc42 ${ }^{\Delta \mathrm{CaaX}} n=86$ cells, CRY2 control $n=85$ cells, WT control $n=246$ cells) and Figure 5A (CRIB-3GFP rga4 $\Delta$ rga6 $\Delta$ : CRY2-Cdc42 ${ }^{\Delta \mathrm{CaaX}} n=83$ cells, CRY2 control $n=80$ cells, WT control $n=145$ cells; Pak1-sfGFP rga4 $\Delta$ rga6 $\Delta$ : CRY2-Cdc42 ${ }^{\Delta \mathrm{CaaX}} n=83$ cells, CRY2 control $n=84$ cells, WT control $n=$ 163 cells).

\subsubsection{Quantifications of the Re-Localization of GFP-Tagged Proteins from Cell Tips}

Scd2-GFP, CRIB-3GFP, Pak1-sfGFP and Scd1-3GFP tip signal analyses were performed from the same time-lapse recordings as cell side re-localization experiments. GFP tip signals were recorded over a 3 pixel-wide by $6-12$ pixel-long $(\approx 0.25 \mu \mathrm{m}$ by $0.5-1 \mu \mathrm{m})$ ROI drawn at the tip of the cells. To derive photobleaching correction coefficients, the average camera background signals $(B c k g)$ from 5 cell-free regions was measured as before, and GFP bleaching from GFP control strain was measured at the cell tip.

$$
\text { Tip GFP bleaching correction coefficient }=\frac{\left(\text { GFP Intensity } y_{t n}-B c k g_{t n}\right)}{\left(\text { GFP Intensit } y_{t 0}-B c k g_{t 0}\right)}
$$

where GFP intensity stands for the signal measured from the tip of GFP control cells, $t_{n}$ represents a given time point along the time course of the experiment and $t_{0}$ represents the initial time point $n=30$ time points). This coefficient was corrected by a moving average smoothing method, as before.

The tip GFP fluorescence intensity values of optogenetic cells was analysed as follows. First, GFP signals was background and bleaching corrected, using Formula (14):

Tip GFP value $_{t n}=\left(\right.$ Tip Raw signal $\left.t_{n}-B_{c k} g_{t n}\right) /$ Tip GFP bleaching correction coef ficient $t_{t n}$ 
where Tip Raw signal intensity represents the GFP raw values at the cell tip, Bckg stands for the average fluorescence intensity of 5 independent cell-free regions and $t_{n}$ represents a given time point along the time course of the experiment ( $n=30$ time points). The tip fluorescence intensities of single optogenetic strains were then normalized relative to their GFP values at the initial time-point.

$$
\text { Normalized tip GFP value }_{t n}=\left(\text { Tip GFP value } \text { tn } / \text { tip }_{\text {GFP }} \text { t0 }\right)
$$

Measurements from cells obtained in 3 experimental replicates are plotted on Figure 1D (CRY2-Cdc42 $2^{\Delta \mathrm{CaaX}} n=88$ cells; CRY2 control $n=87$ cells, WT control $n=146$ cells); Figure 1F (CRY2-Cdc42 $2^{\Delta \mathrm{CaaX}} n=79$ cells; CRY2 control $n=67$ cells, WT control $n=147$ cells): Figure $1 \mathrm{H}$ (CRY2-Cdc42 ${ }^{\Delta \mathrm{CaaX}} n=72$ cells; CRY2 control $n=68$ cells, WT control $n=203$ cells); Figure 2C (Scd2-GFP scd1 $\Delta$ : CRY2-Cdc42 ${ }^{\Delta \mathrm{CaaX}} n=68$ cells, CRY2 control $n=63$ cells, WT control $n=134$ cells; CRIB-3GFP scd1 $\Delta$ : CRY2-Cdc42 ${ }^{\Delta \text { CaaX }} n=65$ cells, CRY2 control $n=67$ cells, WT control $n=131$ cells); Figure 2F (CRY2-Cdc42 ${ }^{\Delta \mathrm{CaaX}} n=69$ cells; CRY2 control $n=80$ cells, WT control $n=160$ cells); Figure 3C (CRY2-Cdc42 $2^{\Delta \mathrm{CaaX}} n=109$ cells; CRY2 control $n=75$ cells, WT control $n=259$ cells) and Figure 3F (CRY2-Cdc42 ${ }^{\Delta \mathrm{CaaX}} n=85$ cells; CRY2 control $n=78$ cells, WT control $n=226$ cells).

\subsubsection{Quantifications of CRIB-3GFP and Cdc42-mCh ${ }^{\mathrm{SW}}$ Relative Expression and Distribution Profiles}

CRIB-3GFP fluorescence intensity was measured from sum projection of 6 Z-stacks (Figure S4B). The background signal from cell-free regions were used to correct the data. The relative fluorescence intensities were calculated by dividing the single-cell CRIB-3GFP fluorescence intensity measurements of WT and Pact1-cdc42-mCh ${ }^{S W}$ cells by the average CRIB-3GFP fluorescence intensity of WT cells.

CRIB-3GFP distribution profiles were generated from sum projection images of 5 middle-sections (Figure S4C). 3-pixel wide ROIs were drawn from side to side following cell membrane contour. The background signal from cell-free regions were used to correct the data. Whole tip profiles were split in half based on the pixel position of their maximum CRIB-3GFP intensity (approximately in the middle of the profile), generating 60 half tips. To take into account the CRIB-3GFP expression level, the average CRIB-3GFP profiles from WT and Pact1-cdc42- $m C h^{S W}$ cells were then normalized by dividing each value by the relative CRIB-3GFP fluorescence intensities values shown in Figure S4B.

\subsubsection{Quantifications of CRIB-3GFP and Pak1-sfGFP at the Cell Sides of WT and rga4trga6 $\Delta$ Mutants}

CRIB-3GFP and Pak1-sfGFP re-localization at the cell sides of WT and rga4 $\Delta r g a 6 \Delta$ mutants were assessed upon $30 \mathrm{~min}$ of photo-activation by recording the average GFP fluorescence intensity over a 3 pixel-wide by 36 pixel-long $(\approx 0.25 \mu \mathrm{m}$ by $3 \mu \mathrm{m})$ ROI drawn parallel to the cell side cortex of CRY2-Cdc42 $2^{\Delta \mathrm{CaOX}}$ and WT control cells. Control GFP strains were imaged in the same pad and analysed in the same way as optogenetic cells: CRIB-3GFP and Pak1-sfGFP fluorescence signals from CRY2-Cdc42 ${ }^{\Delta \mathrm{CaaX}}$ and WT control cells were corrected for the average camera background signals, derived from 6 cell-free regions. The WT control strain, which carries CRIB-3GFP or Pak1-sfGFP like the sample strain of the experiment, but lack the optogenetic system, served as negative control. This controlled that GFP fluorescence changes were not caused by imaging per se. Measurements from these cells were also used as background signal and the average GFP value was subtracted from the the CRY2-Cdc42 ${ }^{\Delta \mathrm{CaaX}}$ measurements for each experimental replicate. Measurements from cells obtained in 3 experimental replicates are plotted on Figure 5C (CRIB-3GFP WT control dark $=146$ cell sides, light $=202$ cell sides; CRIB-3GFP CRY2-Cdc42 ${ }^{\Delta \text { CaaX }}$ dark $=143$ cell sides, light $=207$ cell sides; CRIB-3GFP rga4 $\Delta r g a 6 \Delta$ control dark $=145$ cell sides, light $=209$ cell sides; CRIB-3GFP rga4 $\Delta r g a 6 \Delta$ CRY2-Cdc42 ${ }^{\Delta \mathrm{CaaX}}$ dark $=145$ cell sides, light $=211$ cell sides; Pak1-sfGFP WT control dark $=138$ cell sides, light $=230$ cell sides; Pak1-sfGFP CRY2-Cdc42 ${ }^{\Delta \text { CaaX }}$ dark $=147$ cell sides, light $=221$ cell sides;

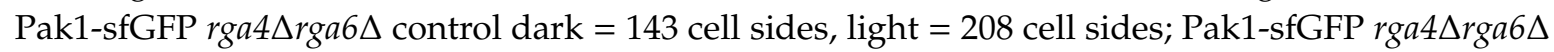
CRY2-Cdc42 ${ }^{\Delta \mathrm{CaaX}}$ dark $=144$ cell sides, light $=222$ cell sides). 


\subsubsection{Cell Size Measurements, Aspect Ratios and Index of Ratios}

The aspect ratio of mutant cells was calculated by dividing the cell length by the cell width (Figure 4A). In Figure 4B, the index of ratios was calculated by dividing CRY2 control aspect ratios by CRY2-Cdc42 4 CaaX and CRY2-Cdc42 ${ }^{\mathrm{Q} 61 \mathrm{~L}, \Delta} \mathrm{CaaX}$.

Figure were assembled with Adobe Photoshop CS5 and Adobe Illustrator CS5. All error bars on bar graphs are standard deviations. For statistical analysis, in Figures 1-3, cumulative GFP signal (addition of GFP signal along the 30 time points of the time-lapse) was calculated from single cell traces of CRY2, CRY2-Cdc42 ${ }^{\Delta \mathrm{CaaX}}$ and GFP control cells.

\subsubsection{Cell Images and Kymographs}

Cell images shown in Figures 1A, 2A-D and 3A-D, and Supplementary Figures S3 and S7 were obtained from time lapses that were first aligned using the StackReg (http://bigwww.epfl.ch/ thevenaz/stackreg/\#Explanations) plugin on image J. Aligned time lapses were corrected for the average camera background by subtracting the average signal from 6 cell-free regions measured over the first frame (t0). Finally, time lapses were corrected for photo-bleaching using the Bleach correction plugin (https://www.embl.de/eamnet/html/bleach_correction.html) on image J. GFP maximum-intensity projections of entire time lapses (30 time points) were used to generate GFP max projection images. GFP max projection images were merged with RFP max maximum-intensity projections, in which the the first frame $(\mathrm{t} 0)$ was omitted in order to segment the plasma membrane. Prior to blue light exposure CRY2-Cdc42 $2^{\Delta \mathrm{CaX}}$ remains cytosolic as shown in Figure S1D.

Kymographs shown in Figure 1B were generated with the MultipleKymograph (https://www. embl.de/eamnet/html/body_kymograph.html) ImageJ plugin. A single pixel-wide ROI was drawn along the cortex at the cell side $(1$ pixel $=0.083 \mu \mathrm{m})$.

\subsubsection{Statistical Analysis}

For statistical analysis, single cell cumulative GFP signals of the entire dataset ( 3 independent experiments combined) were considered, without averaging. For CRIB analysis, we also performed statistical tests on cumulative GFP signal of the second half of the time lapse relative to either the first half or the second half of the CRY2 control, both of which returned statistically significant values ( $p$-values $=0.0008$ and 0.0025 , respectively). By contrast, a comparison of cumulative GFP signal over the first half (first $45 \mathrm{~s}$ ) with the corresponding time lapse in the control was non-significant ( $p$-value $=0.45)$. We also did these comparisons on the Scd1-3GFP signal with similar results (2nd half of CRY2-Cdc $42^{\Delta \mathrm{CaaX}}$ vs. 1st half $p$-value $=0.024 ; 2$ nd half of CRY2-Cdc $42^{\Delta \mathrm{CaaX}}$ vs. 2 nd half of $\mathrm{CRY} 2$ control $p$-value $=0.0035 ; 1$ st half of CRY2-Cdc42 $2^{\Delta \mathrm{CaOX}}$ vs. 1 st half of CRY2 control $p$-value $=$ 0.12). We chose to report the tests on the cumulative signal for the entire time lapse, as these are more conservative, and thus less prone to over-interpretation. Data normality was assessed by the Lilliesfors test and significance by pairwise Kruskal-Wallis analysis. $p$ values show significance of differences between CRY2-Cdc42 ${ }^{\triangle \mathrm{CaaX}}$ and CRY2 cells, unless indicated otherwise. A T-test was used in Figures 4 and 5C. On each box, the central red mark indicates the median, while the bottom and the top edges indicate the 25th and 75th percentiles, respectively. The whiskers extend to the most extreme data points not considering outliers, which are plotted individually using the red ' + ' symbol. All experiments were done at least three independent times. For clarity of the average traces, the plots are shown with standard error of the mean (SEM) in the main figures, which shows the robustness of the average value. The same plots are available in the supplementary figures with standard deviation (SD) to show the biological variability.

Supplementary Materials: The following are available online at http://www.mdpi.com/2073-4409/9/9/2089/s1, Figure S1: Controls for CRY2-Cdc42 ${ }^{\Delta \mathrm{CaOX}}$ optogenetic recruitment, Figure S2: Control and single-cell traces for optogenetic recruitment in WT cells, Figure S3: Additional examples for CRY2-Cdc42 ${ }^{\Delta \mathrm{Ca}}$ induced CRIB-3GFP cell side recruitment, Figure S4: Overexpression of $\mathrm{Cdc} 42-\mathrm{mCh}^{\mathrm{SW}}$ in WT cells does not induce ectopic activation 
of Cdc42, Figure S5: Control and single-cell traces for optogenetic recruitment in scd1 $\Delta$ and gef1 $\Delta$ cells, Figure S6: Control and single-cell traces for optogenetic recruitment of Scd1-3GFP and CRIB-3GFP in WT and sdc2 $\Delta$ cells, Figure S7: Additional examples for CRY2-Cdc42 ${ }^{\Delta \mathrm{CaaX}}$ induced Scd1-3GFP cell side recruitment, Figure S8: Control and single-cell traces for optogenetic recruitment of CRIB-3GFP and Pak1-sfGFP in rga4 $\Delta$ rga6 $\Delta$ cells, Table S1: List of strains used in this study.

Author Contributions: I.L. and S.G.M. conceived the project. N.W. performed the experiments presented in Figure 4. I.L. performed all other experiments. I.L. and S.G.M. wrote the manuscript. S.G.M. acquired funding. All authors have read and agreed to the published version of the manuscript.

Funding: This work was funded by ERC Consolidator grant (CellFusion) to SGM.

Acknowledgments: We thank Aleksandar Vjestica (University of Lausanne) for gift of the CRY2-Cdc42 $2^{\Delta C a a X}$ strain and Serge Pelet (University of Lausanne) for help with MatLab.

Conflicts of Interest: The authors declare no conflict of interest.

\section{References}

1. Goryachev, A.B.; Leda, M. Autoactivation of small GTPases by the GEF-effector positive feedback modules. F1000Research 2019, 8, 1676. [CrossRef] [PubMed]

2. Woods, B.; Lew, D.J. Polarity establishment by Cdc42: Key roles for positive feedback and differential mobility. Small GTPases 2019, 10, 1301-1337. [CrossRef] [PubMed]

3. Martin, S.G. Spontaneous cell polarization: Feedback control of Cdc42 GTPase breaks cellular symmetry. Bioessays 2015, 37, 1193-1201. [CrossRef] [PubMed]

4. Bendezu, F.O.; Vincenzetti, V.; Vavylonis, D.; Wyss, R.; Vogel, H.; Martin, S.G. Spontaneous Cdc42 polarization independent of GDI-mediated extraction and actin-based trafficking. PLoS Biol. 2015, 13, e1002097. [CrossRef]

5. Kelly, F.D.; Nurse, P. Spatial control of Cdc42 activation determines cell width in fission yeast. Mol. Biol. Cell 2011, 22, 38013-38811. [CrossRef]

6. Lamas, I.; Merlini, L.; Vjestica, A.; Vincenzetti, V.; Martin, S.G. Optogenetics reveals Cdc42 local activation by scaffold-mediated positive feedback and Ras GTPase. PLoS Biol. 2020, 18, e3000600. [CrossRef]

7. Endo, M.; Shirouzu, M.; Yokoyama, S. The Cdc42 binding and scaffolding activities of the fission yeast adaptor protein Scd2. J. Biol. Chem. 2003, 278, 8438-8452. [CrossRef]

8. Chang, E.C.; Barr, M.; Wang, Y.; Jung, V.; Xu, H.P.; Wigler, M.H. Cooperative interaction of S. pombe proteins required for mating and morphogenesis. Cell 1994, 79, 1311-1341. [CrossRef]

9. Tay, Y.D.; Leda, M.; Goryachev, A.B.; Sawin, K.E. Local and global Cdc42 GEFs for fission yeast cell polarity are coordinated by microtubules and the Tea1/Tea4/Pom1 axis. J. Cell Sci. 2018. [CrossRef]

10. Vjestica, A.; Zhang, D.; Liu, J.; Oliferenko, S. Hsp70-Hsp40 chaperone complex functions in controlling polarized growth by repressing Hsf1-driven heat stress-associated transcription. PLoS Genet. 2013, 9, e100. [CrossRef]

11. Coll, P.M.; Trillo, Y.; Ametzazurra, A.; Perez, P. Gef1p, a New Guanine Nucleotide Exchange Factor for Cdc42p, Regulates Polarity in Schizosaccharomyces pombe. Mol. Biol. Cell 2003, 14, 313-323. [CrossRef] [PubMed]

12. Hirota, K.; Tanaka, K.; Ohta, K.; Yamamoto, M. Gef1p and Scd1p, the Two GDP-GTP exchange factors for Cdc42p, form a ring structure that shrinks during cytokinesis in Schizosaccharomyces pombe. Mol. Biol. Cell 2003, 14, 36173-36627. [CrossRef] [PubMed]

13. Gallo Castro, D.; Martin, S.G. Differential GAP requirement for Cdc42-GTP polarization during proliferation and sexual reproduction. J. Cell Biol. 2018, 217, 42154-42229. [CrossRef] [PubMed]

14. Das, M.; Wiley, D.J.; Medina, S.; Vincent, H.A.; Larrea, M.; Oriolo, A.; Verde, F. Regulation of cell diameter, For3p localization, and cell symmetry by fission yeast Rho-GAP Rga4p. Mol. Biol. Cell 2007, 18, 2090-2101. [CrossRef] [PubMed]

15. Revilla-Guarinos, M.T.; Martin-Garcia, R.; Villar-Tajadura, M.A.; Estravis, M.; Coll, P.M.; Perez, P. Rga6 is a Fission Yeast Rho GAP Involved in Cdc42 Regulation of Polarized Growth. Mol. Biol. Cell 2016, 27, 1524-1535. [CrossRef] [PubMed]

16. Nakano, K.; Mutoh, T.; Arai, R.; Mabuchi, I. The small GTPase Rho4 is involved in controlling cell morphology and septation in fission yeast. Genes Cells 2003, 8, 357-370. [CrossRef] [PubMed] 
17. Bugaj, L.J.; Choksi, A.T.; Mesuda, C.K.; Kane, R.S.; Schaffer, D.V. Optogenetic protein clustering and signaling activation in mammalian cells. Nat. Methods 2013, 10, 249-252. [CrossRef]

18. Nussinov, R.; Tsai, C.J.; Jang, H. Ras assemblies and signaling at the membrane. Curr. Opin. Struct. Biol. 2020, 62, 1401-1448. [CrossRef]

19. Inouye, K.; Mizutani, S.; Koide, H.; Kaziro, Y. Formation of the Ras dimer is essential for Raf-1 activation. J. Biol. Chem. 2000, 275, 37373-37740. [CrossRef]

20. Kang, P.J.; Beven, L.; Hariharan, S.; Park, H.O. The Rsr1/Bud1 GTPase interacts with itself and the Cdc42 GTPase during bud-site selection and polarity establishment in budding yeast. Mol. Biol. Cell 2010, 21, 3007-3016. [CrossRef]

21. Zhang, B.; Gao, Y.; Moon, S.Y.; Zhang, Y.; Zheng, Y. Oligomerization of Rac1 gtpase mediated by the carboxyl-terminal polybasic domain. J. Biol. Chem. 2001, 276, 89588-89967. [CrossRef] [PubMed]

22. Zhang, B.; Zheng, Y. Negative regulation of Rho family GTPases Cdc42 and Rac2 by homodimer formation. J. Biol. Chem. 1998, 273, 25728-25733. [CrossRef]

23. Remorino, A.; De Beco, S.; Cayrac, F.; Di Federico, F.; Cornilleau, G.; Gautreau, A.; Parrini, M.C.; Masson, J.B.; Dahan, M.; Coppey, M. Gradients of Rac1 Nanoclusters Support Spatial Patterns of Rac1 Signaling. Cell Rep. 2017, 21, 19221-19935. [CrossRef]

24. Maxwell, K.N.; Zhou, Y.; Hancock, J.F. Rac1 Nanoscale Organization on the Plasma Membrane Is Driven by Lipid Binding Specificity Encoded in the Membrane Anchor. Mol. Cell Biol. 2018, 38. [CrossRef] [PubMed]

25. Sartorel, E.; Unlu, C.; Jose, M.; Massoni-Laporte, A.; Meca, J.; Sibarita, J.B.; McCusker, D. Phosphatidylserine and GTPase activation control Cdc42 nanoclustering to counter dissipative diffusion. Mol. Biol. Cell 2018, 29, 1299-1310. [CrossRef]

26. Slaughter, B.D.; Unruh, J.R.; Das, A.; Smith, S.E.; Rubinstein, B.; Li, R. Non-uniform membrane diffusion enables steady-state cell polarization via vesicular trafficking. Nat. Commun. 2013, 4. [CrossRef] [PubMed]

27. Meca, J.; Massoni-Laporte, A.; Martinez, D.; Sartorel, E.; Loquet, A.; Habenstein, B.; McCusker, D. Avidity-driven polarity establishment via multivalent lipid-GTPase module interactions. EMBO J. 2019, 38. [CrossRef]

28. Kennedy, M.J.; Hughes, R.M.; Peteya, L.A.; Schwartz, J.W.; Ehlers, M.D.; Tucker, C.L. Rapid blue-light-mediated induction of protein interactions in living cells. Nat. Methods 2010, 7, 9739-9775. [CrossRef]

29. Wheatley, E.; Rittinger, K. Interactions between Cdc42 and the scaffold protein Scd2: Requirement of SH3 domains for GTPase binding. Biochem. J. 2005, 388, 1771-1784. [CrossRef]

30. Tatebe, H.; Nakano, K.; Maximo, R.; Shiozaki, K. Pom1 DYRK regulates localization of the Rga4 GAP to ensure bipolar activation of Cdc42 in fission yeast. Curr. Biol. 2008, 18, 3223-3230. [CrossRef]

31. Merlini, L.; Khalili, B.; Dudin, O.; Michon, L.; Vincenzetti, V.; Martin, S.G. Inhibition of Ras activity coordinates cell fusion with cell-cell contact during yeast mating. J. Cell Biol. 2018, 217, 1467-1483. [CrossRef]

32. Merlini, L.; Khalili, B.; Bendezu, F.O.; Hurwitz, D.; Vincenzetti, V.; Vavylonis, D.; Martin, S.G. Local Pheromone Release from Dynamic Polarity Sites Underlies Cell-Cell Pairing during Yeast Mating. Curr. Biol. 2016, 26, 1117-1125. [CrossRef] [PubMed]

33. Miller, K.E.; Magliozzi, J.O.; Picard, N.A.; Moseley, J. Sequestration of the exocytic SNARE Psy1 into multiprotein nodes reinforces polarized morphogenesis in fission yeast. BioRxiv 2020. [CrossRef]

34. Dudin, O.; Bendezu, F.O.; Groux, R.; Laroche, T.; Seitz, A.; Martin, S.G. A formin-nucleated actin aster concentrates cell wall hydrolases for cell fusion in fission yeast. J. Cell Biol. 2015, 208, 897-911. [CrossRef] [PubMed]

35. Bähler, J.; Wu, J.Q.; Longtine, M.S.; Shah, N.G.; McKenzie, A., III; Steever, A.B.; Wach, A.; Philippsen, P.; Pringle, J.R. Heterologous modules for efficient and versatile PCR-based gene targeting in Schizosaccharomyces pombe. Yeast 1998, 14, 9439-9451. [CrossRef]

36. Vjestica, A.; Marek, M.; Nkosi, P.J.; Merlini, L.; Liu, G.; Berard, M.; Billault-Chaumartin, I.; Martin, S.G. A toolbox of Stable Integration Vectors (SIV) in the fission yeast Schizosaccharomyces pombe. J. Cell Sci. 2019. [CrossRef]

37. Bendezu, F.O.; Martin, S.G. Actin cables and the exocyst form two independent morphogenesis pathways in the fission yeast. Mol. Biol. Cell 2011, 22, 44-53. [CrossRef]

(C) 2020 by the authors. Licensee MDPI, Basel, Switzerland. This article is an open access article distributed under the terms and conditions of the Creative Commons Attribution (CC BY) license (http://creativecommons.org/licenses/by/4.0/). 